\title{
Anatomical and Gene Expression Analyses of Two Blueberry Genotypes Displaying Differential Fruit Detachment
}

\author{
Tripti Vashisth ${ }^{1}$ \\ Department of Horticulture, 1121 Miller Plant Sciences, University of Georgia, Athens, GA 30602 \\ D. Scott NeSmith ${ }^{2}$ \\ Department of Horticulture, Georgia Station, University of Georgia, Griffin, GA 30223 \\ Anish Malladi ${ }^{3}$ \\ Department of Horticulture, 1121 Miller Plant Sciences, University of Georgia, Athens, GA 30602
}

Additional Index wORDs. abscission, cell wall hydrolysis, fracture plane, fruit harvest, phytohormone signaling

\begin{abstract}
Fruit detachment in blueberry (Vaccinium sp.) may occur through the physiological process of abscission or through physical separation by breakage. Natural and induced fruit detachment through abscission occurs at the peduncle-pedicel junction (PPJ), while detachment through breakage typically occurs at the fruit-pedicel junction (FPJ). The ease of fruit detachment varies across blueberry genotypes, and a better understanding of such variation may allow for the development of genotypes better suited for hand and mechanical harvesting. TH-729 and 'Suziblue' are sibling southern highbush blueberry (hybrids composed largely of Vaccinium corymbosum and Vaccinium darrowi) genotypes derived from the same cross ('Star' $\times$ TH-474) and differ in their fruit detachment characteristics. Anatomical and molecular basis of the difference in fruit detachment between these genotypes was investigated in this study. Greater than $85 \%$ of the mature fruit of $\mathrm{TH}-729$ detached at the PPJ in response to mechanical shaking in contrast to that observed in 'Suziblue', where greater than $90 \%$ of the fruit detached at the FPJ. The anatomy of the abscission zones (AZs) at the PPJ was similar between the two genotypes indicating that they did not differ in the establishment of the AZ. The fracture plane at the PPJ of manually detached fruit was more even in TH-729 compared with that in 'Suziblue', where many ruptured cells were evident. These data suggest advanced progression of abscission at the PPJ in TH-729 compared with that in 'Suziblue'. The expression of 28 genes related to cell wall and membrane metabolism, phytohormone metabolism and signaling, and transcriptional regulation was compared between the two genotypes. Of these, two genes, ILL1 (iaa-leu resistant 1 like 3) and BIMI (bes-interacting myc like1), associated with auxin metabolism and brassinosteroid signaling displayed over 3-fold and 1.5-fold higher transcript accumulation, respectively, in TH-729. Also, OPR1 (12-oxophytodienoate reductase), a gene associated with jasmonate (JA) biosynthesis, displayed 33\% lower transcript levels in TH-729. As phytohormone signaling regulates the acquisition of competence for abscission, these data suggest that this phase of abscission progression at the PPJ differed between the two genotypes. Together, data from this study suggest inherent differences in the progression of abscission at the PPJ in blueberry. Such variation can be exploited to develop genotypes with desired harvesting characteristics.
\end{abstract}

Blueberry is a fruit crop of rapidly emerging economic significance in the United States. Harvesting practices during blueberry production can be improved through a better understanding of the process of fruit detachment. Mature fruit detachment in blueberry can occur at one of two locations (Fig. 1). Fruit detachment by abscission in blueberry generally occurs at the PPJ, while detachment at the FPJ is primarily due to breakage (Vashisth and Malladi, 2013, 2014). Natural, wound-induced, and plant growth regulator-induced mature fruit abscission occurs at the PPJ (Vashisth and Malladi, 2013, 2014). Harvesting fruit by hand and the majority of mechanical harvesting in blueberry are typically associated with detachment at the FPJ (Howell et al., 1976; Malladi et al., 2013;

Received for publication 9 Sept. 2015. Accepted for publication 9 Oct. 2015. This research was supported by a grant from the U.S. Department of Agriculture, Specialty Crops Research Initiative 2008-51180-19579. We thank Lisa K. Johnson for help with experiments in this study.

${ }^{1}$ Assistant professor. Current address: 700 Experiment Station Road, Citrus Research and Education Center, Institute of Food and Agricultural Sciences, University of Florida, Lake Alfred, FL 33850.

${ }^{2}$ Professor.

${ }^{3}$ Corresponding author. E-mail: malladi@uga.edu.
Takeda et al., 2008; Vashisth and Malladi, 2013). Fruit detachment at the FPJ is preferable for fresh fruit as detachment at the PPJ results in "stemmy" fruit that retain the pedicel. Retention of the pedicel on the fruit is considered to be a defect that reduces fruit quality and the presence of greater than $10 \%$ of such fruit precludes their classification as U.S. No. 1 grade (U.S. Department of Agriculture, 1995). While intuitively it is known that blueberry genotypes differ in the ease of fruit detachment, understanding the morphological, anatomical, physiological, and molecular bases of such differences in fruit detachment across blueberry genotypes can potentially aid in the development of cultivars with reduced detachment at the PPJ, which makes them better suited for hand and mechanical harvesting.

Organ detachment can occur either through breakage and separation from the plant or due to abscission. While breakage generally occurs along a weak point between the organ and the plant, abscission is a developmentally and physiologically regulated process that involves the separation of the organ from the plant in a programmed manner or in response to external stimuli. Abscission occurs at specific regions termed abscission zones. The progression of organ abscission in plants has been divided into four main phases (Estornell et al., 2013; 


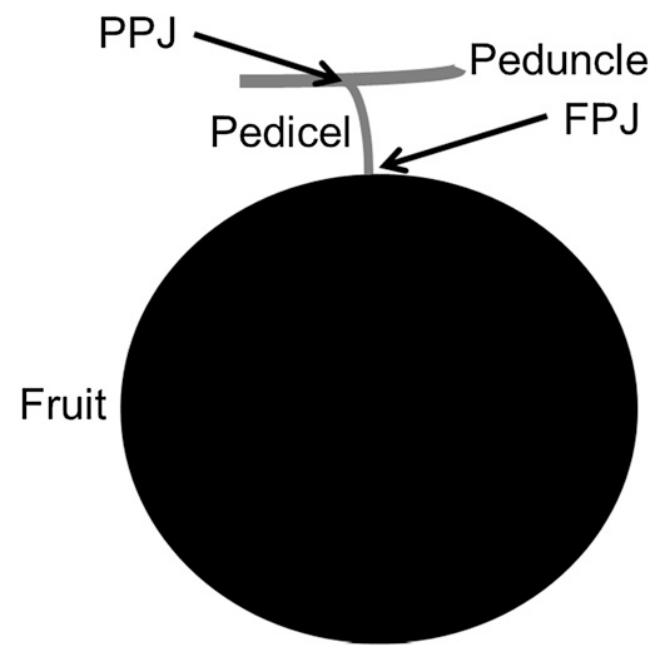

Fig. 1. Fruit detachment points in blueberry. The two potential fruit detachment points of the blueberry fruit are illustrated; PPJ = peduncle-pedicel junction, $\mathrm{FPJ}=$ fruit-pedicel junction.

Kim, 2014; Patterson, 2001). The first stage of abscission involves the establishment of the $\mathrm{AZ}$ and is associated with cell differentiation leading to the development of multiple cell layers, including a separation layer between the organ destined for detachment and the plant. Several genes that control the establishment of the AZ such as jointless and blade on petiole have been identified in tomato (Solanum lycopersicum) and arabidopsis (Arabidopsis thaliana) (Mao et al., 2000; McKim et al., 2008). Establishment of the AZ is followed by a phase wherein competence for abscission is attained through developmental and phytohormone signaling (Taylor and Whitelaw, 2001). Ethylene and auxin have been implicated extensively in regulating the competence and thereby the timing of abscission (Estornell et al., 2013; Kim, 2014; Patterson, 2001; Taylor and Whitelaw, 2001). While ethylene accelerates the progression of abscission, consistent auxin flux through the AZ inhibits it. The balance between the relative quantities and signaling of these phytohormones, and their interactions with external stimuli, may regulate the timing and progression of abscission (Estornell et al., 2013; Taylor and Whitelaw, 2001). Recently other phytohormones such as JAs have also been implicated in the regulation of this phase of organ abscission. Jasmonates may accelerate the progression of floral and fruit abscission, potentially through ethylene-dependent and -independent mechanisms (Hartmond et al., 2000; Kim et al., 2013; Malladi et al., 2012). Acquisition of competence for abscission is followed by the activation of cell separation leading to organ detachment. This phase is associated with various morphological and biochemical changes within the AZ cells, including alterations in cell wall composition, a process mediated by a set of enzymes such as polygalacturonases, cellulases, xyloglucan endo-transglycosylases/hydrolases, and expansins (reviewed in Kim, 2014 and Taylor and Whitelaw, 2001). The activity of these enzymes can lead to the dissolution of the cell wall aiding in the progression of cell separation. Various transcription factors are involved in coordinating the progression through this phase of organ abscission (Estornell et al., 2013; Kim, 2014). Also, recent studies have identified multiple components of a signal transduction pathway regulating this phase during floral organ abscission in arabidopsis
(Liljegren, 2012). The final phase that can occur in conjunction with organ detachment involves repair at the point of detachment and formation of a protective layer (Patterson, 2001). Although much has been learned from various model systems such as arabidopsis and tomato, information on genes that regulate these phases of organ abscission in fruit crops such as blueberry is currently limited. Moreover, the potential roles of such genes in facilitating differential fruit detachment across different genotypes are not clearly understood.

The southern highbush blueberry cultivar, Suziblue, was evaluated previously as TH-730 and commercially released as an early-season cultivar with favorable fruit characteristics (NeSmith, 2010). TH-729 is an unreleased sibling of 'Suziblue'. Both these genotypes were selected from a cross between 'Star' and TH-474 (NeSmith, 2010). Field evaluations of TH-729 indicated that its mature fruit easily detach in response to gentle touch or shaking. Preliminary studies also indicated that mature fruit detachment in TH-729 primarily occurred at the PPJ, in contrast to 'Suziblue', which generally detached at the FPJ when harvested by hand. These observations suggested a difference in fruit detachment between the two genotypes. Such variation may be due to inherent differences in the establishment of the AZ, differences in the timing of acquisition of competence for abscission, or differences in the progression of the cell separation process. Comparative analyses of these genotypes may increase our understanding of the physiology of blueberry fruit abscission and may aid in developing tools to improve harvesting practices. Hence, the objective of this study was to determine the basis of differences in fruit detachment between TH-729 and 'Suziblue'. Fruit detachment in response to mechanical shaking, anatomical characterization of the AZs, and differential gene expression between the two genotypes was studied to address this objective.

\section{Materials and Methods}

Fruit detachment in RESPONSE TO MEChANICAL SHAKING. Container grown TH-729 and 'Suziblue' plants $(n=5)$ maintained at the Griffin Experiment Station, University of Georgia, Griffin, GA, were used to study fruit detachment characteristics in response to mechanical shaking. The plants were grown outside in 11.3-L containers to allow for chilling accumulation and were brought into the greenhouse set at day/night temperatures of $18.3 / 29.4{ }^{\circ} \mathrm{C}$ after pollination and fruit set in Apr. 2013. A handheld mechanical shaker (Malladi et al., 2013) was used to perform mechanical shaking in June 2013. The mechanical shaker consisted of a variable-speed reciprocating saw fitted with a stainless steel adapter that could be clamped on to a branch for shaking. All plants had mature fruit (blue/black color) at the time of the experiment. The adapter of the mechanical shaker was attached to the branch several inches below the fruit and shaken for $5 \mathrm{~s}$. The number of fruit was counted before and after mechanical shaking and used to calculate percent fruit detachment. Detached fruit were collected in a catch frame placed below the branch. From the collected fruit, the proportion of mature fruit (blue/black fruit at harvestable maturity) that detached along with the pedicel was determined (Fig. 1).

Light MiCROSCOPY. TH-729 and 'Suziblue' plants $(n=4)$ grown at the Blueberry Research Farm, University of Georgia, Alapaha, were used for the light microscopy analysis as well as the scanning electron microscopy (SEM) and gene expression analyses described below. For light microscopy, the AZs at the 
PPJ (Fig. 1) along with the pedicel were manually dissected from mature fruit and immediately fixed in 10 formaldehyde : 5 acetic acid : 50 ethanol. Two fruit per replicate were used for this analysis. The samples were rinsed in deionized water, dehydrated in a graded series of ethanol, embedded in paraffin, and sectioned $(10 \mu \mathrm{m})$ using a microtome as described in Vashisth and Malladi (2013). The sections were stained in a 1\% aqueous solution of toluidine blue and visualized under a microscope.

SCANNING ELECTRON Microscopy (SEM). For SEM, the pedicels were separated manually from the peduncle or from the mature fruit to obtain the PPJ and the FPJ fracture planes (Fig. 1), respectively $(n=4)$. These samples were immediately fixed in 5\% glutaraldehyde : $0.1 \mathrm{M}$ potassium phosphate buffer (1:1), stored at $4{ }^{\circ} \mathrm{C}$, and used for SEM analysis. The pedicel ends of the detached PPJ and FPJ were analyzed. Samples were processed for SEM as described in Vashisth and Malladi (2013). Briefly, the samples were cut into $\approx 1-2 \mathrm{~mm}$ sections and rinsed thrice with the fixative buffer for $15 \mathrm{~min}$. The samples were immediately treated with osmium tetroxide for $2 \mathrm{~h}$ and rinsed. The rinsed samples were dehydrated through a graded ethanol series. Ethanol was replaced by liquid $\mathrm{CO}_{2}$, which was brought to the critical point in a critical point dryer (Autosamdri-814; Tousimis Research Corp., Rockville, MD). Subsequently, samples mounted on aluminum stubs were coated with gold in a sputter coater and carbon coater (SPI Supplies/Structure Probe, West Chester, PA). Samples were observed using a scanning electron microscope (1450EP; Carl Zeiss MicroImaging, Thornwood, NY).

Gene EXPRESSION. The PPJ tissues from $\approx 50$ mature fruit were dissected manually, pooled within each replicate $(n=4)$, immediately frozen in liquid nitrogen, and stored until further analysis. About $1 \mathrm{~mm}$ or less of adjacent tissue was attached to the dissected AZs. RNA extraction, cDNA synthesis, and gene expression profiling were performed as described by Vashisth et al. (2011) with some modifications. Briefly, $0.1 \mathrm{~g}$ of the sample was ground to fine powder and added to $0.75 \mathrm{~mL}$ of the extraction buffer. The RNA extraction buffer consisted of $2 \%$ cetyltrimethylammonium bromide, $2 \%$ polyvinylpyrrollidone, $100 \mathrm{~mm}$ Tris- $\mathrm{HCl}, 25 \mathrm{~mm}$ ethylenediaminetetraacetic acid, $2 \mathrm{M}$ sodium chloride, $3.44 \mathrm{~mm}$ spermidine, and $2 \% \beta$-mercaptoethanol. All solutions were prepared using $0.1 \%$ diethylpyrocarbonate (DEPC) treated water. The buffer was warmed to $65^{\circ} \mathrm{C}$ before the addition of the ground tissue. The sample-buffer slurry was mixed briefly and incubated at $65^{\circ} \mathrm{C}$ for $10 \mathrm{~min}$, mixed again, and incubated at room temperature for $5 \mathrm{~min}$. The mixtures were extracted twice with an equal volume of chloroform : isoamyl alcohol (24:1) with centrifugation at $4{ }^{\circ} \mathrm{C}$ for $15 \mathrm{~min}$ $\left(5000 g_{\mathrm{n}}\right)$. The supernatant was transferred to sterile tubes followed by the addition of 0.25 volumes of lithium chloride $(10 \mathrm{M})$. The samples were mixed gently and precipitated overnight at $4{ }^{\circ} \mathrm{C}$. Samples were subsequently centrifuged at $4{ }^{\circ} \mathrm{C}$ for $20 \mathrm{~min}\left(12,000 \mathrm{~g}_{\mathrm{n}}\right)$. After discarding the supernatant, the RNA pellets were washed twice with $500 \mu \mathrm{L}$ of $70 \%$ ethanol, dissolved in DEPC and stored at $-80^{\circ} \mathrm{C}$. Before cDNA synthesis, potential genomic DNA contamination was removed by treating $1 \mu \mathrm{g}$ of the total RNA with DNase $\left[37^{\circ} \mathrm{C}\right.$ for $34 \mathrm{~min}$ (Promega Corp., Madison, WI)]. The DNase-treated RNA was reverse transcribed using ImProm-II Reverse Transcriptase (Promega Corp.) and oligo(dT) in a $20 \mu \mathrm{L}$ reaction volume. Reverse transcription was performed at $42{ }^{\circ} \mathrm{C}$ for $75 \mathrm{~min}$, followed by incubation at $75^{\circ} \mathrm{C}$ for $15 \mathrm{~min}$. The cDNA samples were subsequently diluted 10 -fold and stored at $-20{ }^{\circ} \mathrm{C}$ until further analysis. Gene expression analysis was performed using quantitative real-time polymerase chain reaction (qRT-PCR). Twenty-eight genes related to cell wall and membrane metabolism, phytohormone metabolism and signaling, and transcriptional regulation were identified and selected from sequencing data generated using the Roche 454 (454 Life Sciences, Branford, CT) and Illumina (San Diego, CA) sequencing platforms from rabbiteye $(V$. ashei) and southern highbush blueberry AZ tissues. Genes were selected from the next generation data sets based on their putative functions and/or their differential expression during fruit detachment (T. Vashisth, L.K. Johnson, and A. Malladi, unpublished data). The list of genes, their arabidopsis homologs, and primers used in this study are presented in Supplemental Table 1, and nucleotide sequences of the genes are presented in Supplemental Table 2. Primers were designed either manually or using PrimerQuest (Integrated DNA Technologies, Coralville, IA). Previous analysis indicated that gene sequences from different types of blueberry generally display high levels of identity and that primers designed from one Vaccinium species would be generally applicable to others (Vashisth et al., 2011). All qRT-PCR reactions were performed using a real-time PCR system (Stratagene Mx3005P; Agilent Technologies, Santa Clara, CA). A reaction volume of $12 \mu \mathrm{L}$ with $1 \mu \mathrm{L}$ of diluted cDNA and $6 \mu \mathrm{L}$ of $2 \mathrm{X}$ Veriquest SYBR Green Master Mix (Affymetrix, Santa Clara, CA) was used. The thermal cycling conditions were the following: $95^{\circ} \mathrm{C}$ for $10 \mathrm{~min}, 40$ cycles of $94{ }^{\circ} \mathrm{C}$ for $30 \mathrm{~s}$, and $60{ }^{\circ} \mathrm{C}$ for $1 \mathrm{~min}$. Melt-curve analysis was performed at the end of the qRT-PCR reactions to control primer specificity. Control reactions without a template and without the reverse transcriptase were performed for each gene. The primer efficiency $(E)$ for each gene was determined using LinRegPCR (Ramakers et al., 2003; Ruijter et al., 2009). Relative expression was calculated using a modified Pfaffl method (Pfaffl, 2001) and as described in Rieu and Powers (2009). Relative quantity (RQ) for each sample was calculated using the formula, $1 / E^{\mathrm{Cq}}$, where $\mathrm{Cq}$ is the quantification cycle (threshold cycle). The RQ was normalized using $R N A$ helicase-like 8 (RH8) and clathrin adaptor complexes medium subunit family protein a (CACSa) as these were previously found to be among the most stable reference genes in blueberry (Vashisth et al., 2011). The geometric mean of expression of these two reference genes (normalization factor) was used for normalization.

StatistiCAL ANALYSES. All statistical analyses were performed using SigmaPlot 11 (Systat Software, San Jose, CA). Percent fruit detachment with the pedicel attached was compared between TH-729 and 'Suziblue' using the Student's $t$ test ( $n=5, \alpha=0.05$ ). For gene expression data, the normalized RQ values were $\log _{2}$ transformed before statistical analyses. The Student's $t$ test was used for analyzing gene expression data ( $n=4, \alpha=0.05$ ), and the Mann-Whitney rank-sum test was applied if the data did not meet the assumptions of equal variance or normal distribution.

\section{Results and Discussion}

Fruit detachment Characteristics. Mechanical shaking for $5 \mathrm{~s}$ resulted in a similar extent of mature fruit detachment in TH-729 and 'Suziblue'. Greater than $85 \%$ of mature fruit detachment in TH-729 occurred at the PPJ (Fig. 2). In comparison, only $\approx 9 \%$ of the mature fruit detached at this location in 'Suziblue', while the rest detached at the FPJ. These data clearly indicate that mature fruit detachment in TH-729 


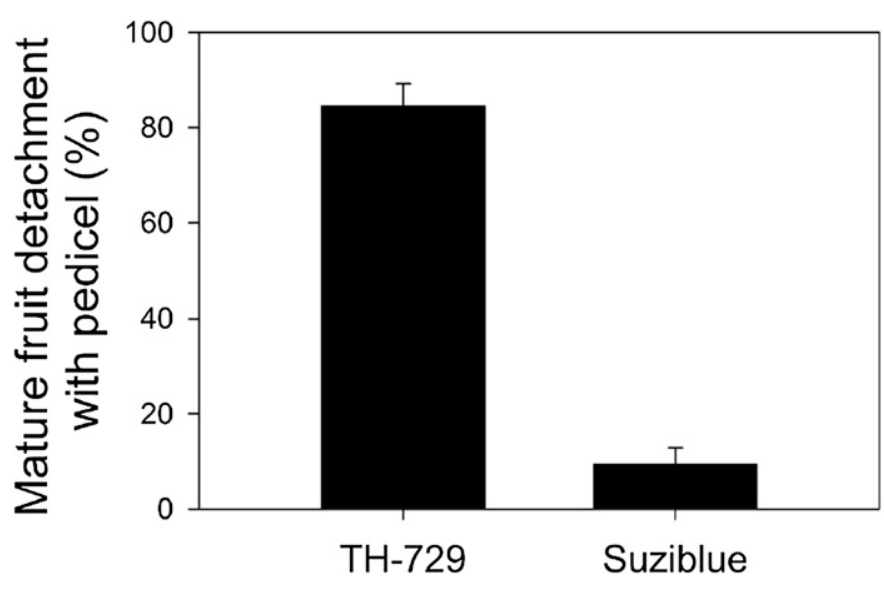

Fig. 2. Mature fruit detachment in response to mechanical shaking in the southern highbush blueberry genotypes, TH-729 and 'Suziblue'. Mechanical shaking was performed for $5 \mathrm{~s}$, the detached fruit were collected in a catch frame and the proportion of mature fruit with the pedicel attached was determined. Error bars indicate the standard error of the mean $(n=5)$. Percent fruit detachment at the peduncle-pedicel junction was significantly different between the two genotypes $(P<0.001$; Student's $t$ test $)$.

occurred preferentially at the PPJ to a much greater extent than that in 'Suziblue' $(P<0.001)$. A preliminary analysis with fieldgrown plants also indicated a higher propensity for TH-729 mature fruit to detach at the PPJ (data not shown). The extent of fruit detachment at the FPJ in 'Suziblue' is similar to that noted in previous reports of fruit detachment in response to mechanical shaking in blueberry (Malladi et al., 2013; Vashisth and Malladi, 2013). Such detachment at the FPJ is due to physical breakage, while fruit detachment at the PPJ is aided by the progression of abscission (Vashisth and Malladi, 2013). It is likely that enhanced loosening at the PPJ in TH-729 resulted in a higher extent of fruit detachment at this location in response to mechanical shaking.

Anatomy of The AZ. The AZs at PPJ were anatomically similar in TH-729 and 'Suziblue' (Fig. 3). Multiple layers of small and round cells were observed on either side of the vascular tissue within the AZ at the PPJ in TH-729 and 'Suziblue', consistent with the blueberry fruit AZ anatomy described previously (Vashisth and Malladi, 2013), and that described in other plant systems (Roberts et al., 2002; Sexton and Roberts, 1982). A clear line of fracture within the AZ was not evident in either of the two genotypes. These data suggest that differences in fruit detachment at the PPJ between the two genotypes were not associated with differences in the development or establishment of the AZ.

Fracture Plane MORPhOlogy. The pedicel end of the PPJ in TH-729 generally displayed an even fracture plane with some minor disruption of cells (Fig. 4A). Small and rounded cells were visible at the surface and at the periphery of the fracture plane. Cell rupture and breakage, and some tearing of the tissues were observed at the center of the fracture plane corresponding to the region with the vascular tissues. These observations are consistent with previous descriptions of natural progression of abscission at the PPJ during fruit detachment and progression of abscission in response to the application of abscission agents such as methyl jasmonate (MeJA) and ethephon in rabbiteye blueberry (Vashisth and Malladi, 2013). In 'Suziblue', the fracture plane at the PPJ displayed extensive disruption of cells and a highly uneven

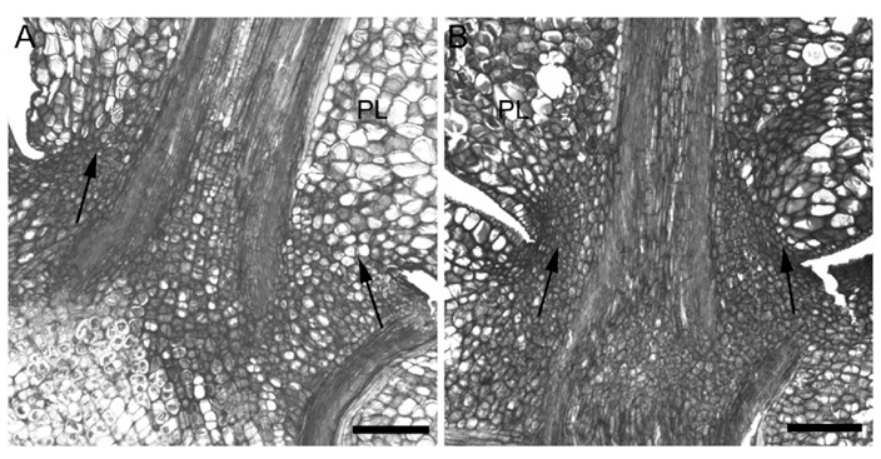

Fig. 3. Anatomy of the abscission zone $(A Z)$ at the peduncle-pedicel junction (PPJ) in the southern highbush blueberry genotypes, TH-729 and 'Suziblue'. The mature fruit AZ at the PPJ in (A) TH-729 and (B) 'Suziblue' are shown. The arrows in each panel point to the AZ at the PPJ; scale bar $=200 \mu \mathrm{m}$, $\mathrm{PL}=$ pedicel.

surface indicating tearing of tissues (Fig. 4B). Extensive disruption of cells along the fracture plane may be indicative of slower progression of developmental abscission and cell separation at the PPJ in 'Suziblue'. In both genotypes, the fracture plane at the pedicel end of the FPJ contained parts of the fruit tissue in addition to extensively disrupted cells (Fig. 4C and D). These data indicate that fruit detachment at the FPJ involved breakage of cells within this junction, consistent with a previous report of similar tissue disruption at the FPJ in rabbiteye blueberry (Vashisth and Malladi, 2013). Together, the SEM analyses indicate that advanced progression of abscission at the PPJ in TH-729 allowed for less disruptive fruit detachment at this location in comparison with that in 'Suziblue'.

Differential GeNe EXPRESSION. The expression of 28 genes related to cell wall and membrane metabolism, phytohormone metabolism and signaling, and transcriptional regulation was compared in the PPJ tissues of TH-729 and 'Suziblue'. Three genes were differentially expressed between the two genotypes (Fig. 5). Interestingly, all these genes were potentially associated with phytohormone metabolism or signaling, specifically that of auxins, JAs, and brassinosteroids.

A gene associated with auxin homeostasis (ILL1) displayed over 3-fold higher transcript accumulation in TH-729 compared with that in 'Suziblue' (Fig. 5). Ethylene and auxin are key phytohormones regulating the progression of abscission (Estornell et al., 2013; Taylor and Whitelaw, 2001). Auxin flux through the AZ negatively regulates the progression of abscission while increase in ethylene promotes it. In arabidopsis, ILL3 and similar genes function as amido-hydrolases, which cleave the amino acid (typically leucine or phenylalanine) conjugated to indole acetic acid (IAA) to release the free and biologically active form of the auxin (Bartel and Fink, 1995; Davies et al., 1999). Accelerated progression of abscission in TH-729 may be associated with a reduction in auxin flux through the AZ. Release of free auxin from conjugated forms at the AZ may occur in response to such a decrease in auxin flux. It is possible that such release of free auxins is mediated by higher accumulation of ILL1 transcripts in TH-729. Increase in the expression of a similar gene, ILR, in the tomato flower AZ was also noted previously in response to flower removal, a treatment that decreases auxin flux through the $\mathrm{AZ}$ and accelerates abscission (Meir et al., 2010). Also, other genes associated with release of free IAA from storage forms were upregulated in tomato pedicel AZs during abscission (Nakano et al., 2013). 

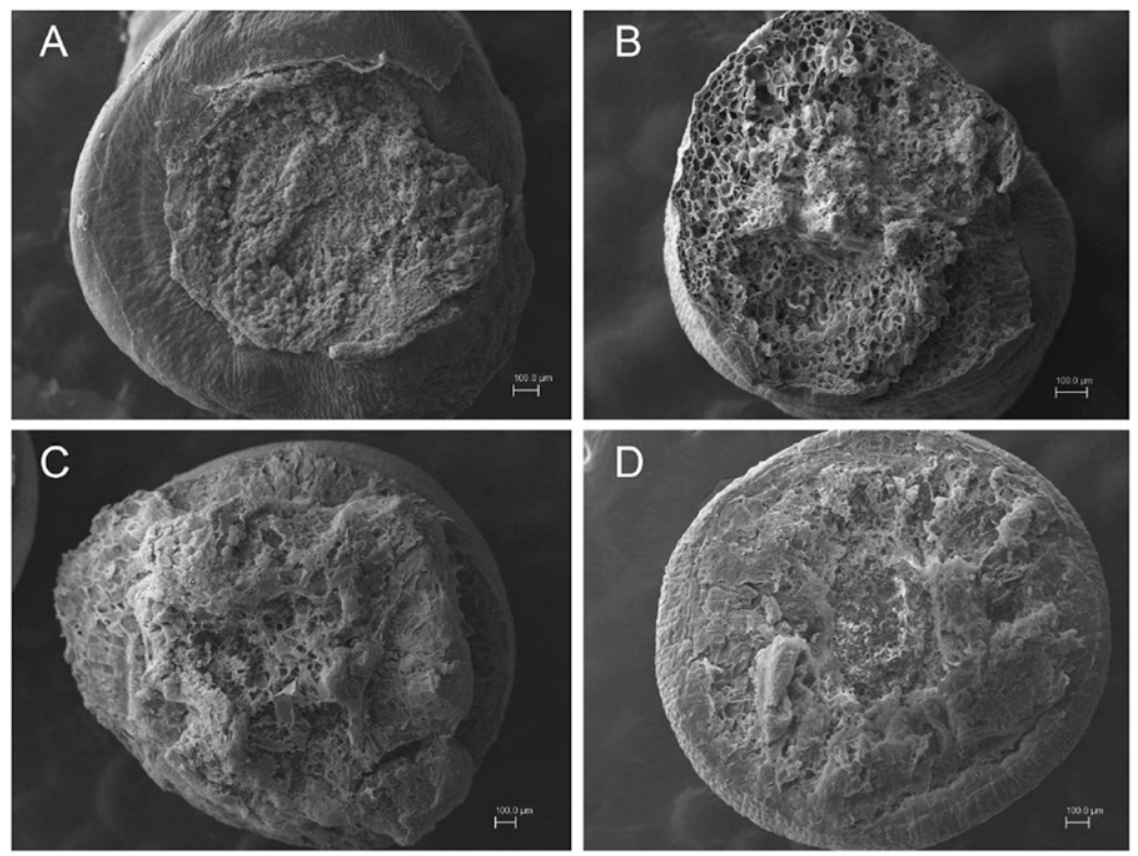

Fig. 4. Analysis of the abscission zone (AZ) fracture planes in the southern highbush blueberry genotypes: (A and C) TH-729 and (B and D) 'Suziblue'. The mature fruit AZs at the (A and B) peduncle-pedicel junction were manually separated and the fracture plane at the pedicel end was analyzed using scanning electron microscopy. Similarly, the pedicel was manually separated from mature fruit at the $(\mathbf{C}$ and $\mathbf{D})$ fruit-pedicel junction and the fracture plane at the pedicel end was analyzed; scale bar $=100 \mu \mathrm{m}$.

The JA biosynthesis-related enzyme, 12-oxophytodienoate reductase (OPR) catalyzes the conversion of 12-oxophytodienoic acid to 3-oxo-2-(2-pentenyl)-cyclopentane-1-octanoic acid, which subsequently undergoes multiple $\beta$-oxidation steps to yield jasmonic acid (Wasternack, 2007). A gene putatively coding for an OPR in blueberry, OPR1, displayed 33\% lower transcript levels in TH-729 compared with that in 'Suziblue' (Fig. 5). Several studies have implicated JAs in the regulation of abscission in various fruits such as sweet orange (Citrus sinensis), grape (Vitis vinifera), olive (Olea europaea), and melon (Cucumis melo) (Corbacho et al., 2013; Fidelibus et al., 2007; Hartmond et al., 2000; Parra et al., 2013). Recently, an arabidopsis mutant displaying delayed floral organ abscission was found to be allelic to coronatine insensitive 1 (COI1), a coreceptor involved in JA responses (Kim et al., 2013). Also in blueberry, application of MeJA resulted in effective and rapid detachment of mature fruit (Malladi et al., 2012; Vashisth and Malladi, 2013, 2014). The above studies indicate a potentially important role for JAs in promoting the physiological progression of organ abscission. Phytohormone biosynthesis and signaling including those of JAs are often subject to positive and negative feedback regulation (Kazan and Manners, 2008). Considering that external applications of MeJA promote blueberry abscission (Malladi et al., 2012; Vashisth and Malladi, 2013), it may be possible that progression of abscission involves increased synthesis of bioactive JAs, which may in turn downregulate components of their biosynthesis pathway to facilitate fine regulation of JA concentration. Lower transcript levels of OPR1 in TH-729 may reflect such a negative feedback regulatory mechanism.

The transcript levels of a component of brassinosteroid signaling, BIM1, was greater than 1.5-fold higher in TH-729. BIM1 encodes a basic loop-helix-loop type of transcription factor that interacts with other transcription factors such as BES1 to bind to the promoter regions of brassinosteroid-regulated genes and modulate their expression (Yin et al., 2005). Although, the direct role of brassinosteroids in regulating abscission is unclear, they are known to regulate the expression of multiple genes associated with cell wall modification (Sun et al., 2010) and were previously found to be associated with tomato abscission (Nakano et al., 2013). Particularly, recent studies suggest that a brassinosteroid feedback regulatory mechanism is involved in cell wall homeostasis through the modulation of pectin methylesterases (PMEs) (Wolf et al., 2012). PMEs have long been known to be involved in cell wall alteration during organ abscission (Agusti et al., 2008, 2009; Cai and Lashbrook, 2008). Additionally, an in silico analysis suggests that BIM1 may interact with MYC2, a transcription factor that functions as a master regulator of JA signaling suggesting cross-talk between JA and brassinosteroid signaling (Chen et al., 2012; Kazan and Manners, 2013). Hence, it may be speculated that enhanced BIM1-mediated brassinosteroid signaling and its potential interaction with JA signaling in TH-729 are associated with accelerated progression of abscission.

Together, data from this study indicate that genes associated with phytohormone metabolism and signaling were differentially expressed between the two genotypes. Among the different phases of abscission, phytohormone metabolism and signaling generally affect the second phase of abscission involving acquisition of competence (Estornell et al., 2013; Kim, 2014). In fact, mutants with defects in phytohormone metabolism or signaling often display differences in the timing of abscission (Estornell et al., 2013; Kim, 2014). Hence, it is possible that TH729 and 'Suziblue' differ in their fruit detachment characteristics due to altered timing of acquisition of competence for abscission. It is possible that such competence for abscission is acquired developmentally earlier in TH-729. None of the genes associated with cell wall or membrane metabolism studied here were significantly different in their expression across the two genotypes. It is likely that similar genes not represented in this study are involved in the differential progression of cell separation between TH-729 and 'Suziblue'. A comprehensive transcriptomics analysis is essential to address this possibility.

\section{Conclusions}

Sibling southern highbush blueberry genotypes TH-729 and 'Suziblue' displayed clear differences in the propensity for fruit detachment at the PPJ. These genotypes did not differ in the establishment of the AZ at the PPJ but were likely affected in the progression of abscission at this location. Genes associated primarily with phytohormone metabolism and signaling were 

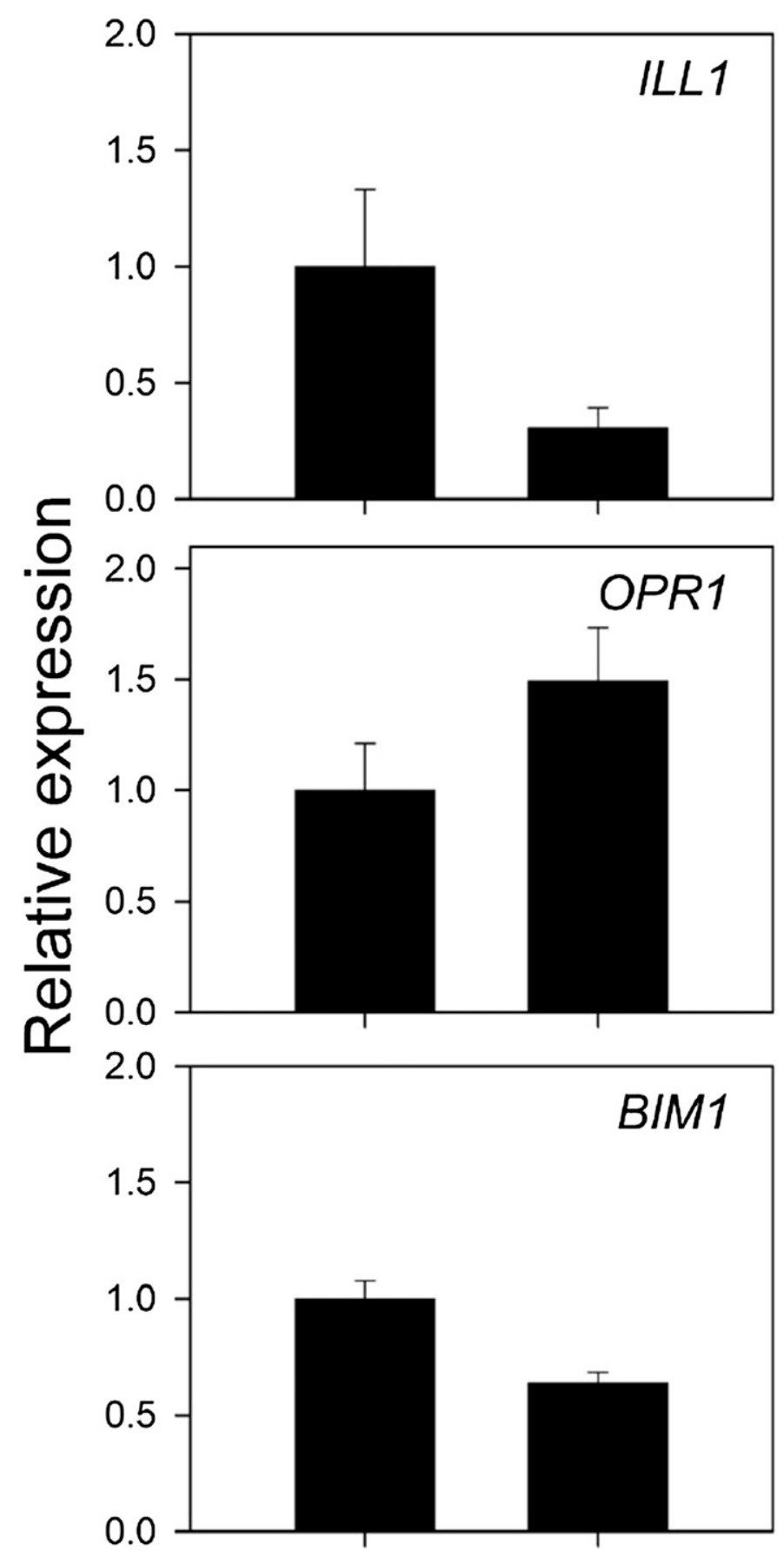

\section{TH-729 Suziblue}

Fig. 5. Differential gene expression between the southern highbush blueberry genotypes, TH-729 and 'Suziblue', at the abscission zone. Gene expression was determined using quantitative real-time polymerase chain reaction and normalized to the expression of $\mathrm{RH} 8$ and $\mathrm{CACS} a$. Gene expression relative to the average expression of the given gene in TH-729 is presented. Error bars indicate the standard error of the mean $(n=4)$. The displayed genes significantly differed in expression between the two genotypes $(P<0.05$; Student's $t$ test).

differentially expressed between the two genotypes, suggesting that they may differ in the timing of acquisition of competence for abscission. If this is the case, the three differentially expressed genes and the mechanisms they influence provide a good starting point to further evaluate the genetic and physiological factors that regulate differences in fruit detachment between the two genotypes. Differences in the ease of fruit detachment across blueberry genotypes as demonstrated in this study indicates that this trait can be selected through breeding and other approaches to develop cultivars with better hand and mechanical harvesting characteristics.

\section{Literature Cited}

Agusti, J., P. Merelo, M. Cercos, F. Tadeo, and M. Talon. 2008. Ethylene-induced differential gene expression during abscission of citrus leaves. J. Expt. Bot. 59:2717-2733.

Agusti, J., P. Merelo, M. Cercos, F. Tadeo, and M. Talon. 2009. Comparative transcriptional survey between laser-microdissected cells from laminar abscission zone and petiolar cortical tissue during ethylene-promoted abscission in citrus leaves. BMC Plant Biol. 9:127.

Bartel, B. and G.R. Fink. 1995. ILR1, an amidohydrolase that releases active indole-3-acetic acid from conjugates. Science 268:1745-1748.

Cai, S. and C.C. Lashbrook. 2008. Stamen abscission zone transcriptome profiling reveals new candidates for abscission control: Enhanced retention of floral organs in transgenic plants overexpressing Arabidopsis ZINC FINGER PROTEIN2. Plant Physiol. 146:1305-1321.

Chen, Y.-A., Y.-C. Wen, and W.-C. Chang. 2012. AtPAN, an integrated system for reconstructing transcriptional regulatory networks in Arabidopsis thaliana. BMC Genomics 13:85.

Corbacho, J., F. Romojaro, J.-C. Pech, A. Latche', and M.C. GomezJimenez. 2013. Transcriptomic events involved in melon maturefruit abscission comprise the sequential induction of cell-wall degrading genes coupled to a stimulation of endo and exocytosis. PLoS One 8:e58363, doi: 10.1371/journal.pone.0058363.

Davies, R., D. Goetz, J. Lasswell, M. Anderson, and B. Bartel. 1999. IAR3 encodes an auxin conjugate hydrolase from Arabidopsis. Plant Cell 11:365-376.

Estornell, L.H., J. Agustí, P. Merelo, M. Talón, and F.R. Tadeo. 2013. Elucidating mechanisms underlying organ abscission. Plant Sci. 199-200:48-60.

Fidelibus, M.W., K.A. Cathline, and J.K. Burns. 2007. Potential abscission agents for raisin table and wine grapes. HortScience 42:1626-1630.

Hartmond, U., R. Yuan, J.K. Burns, A. Grant, and W.J. Kender. 2000. Citrus abscission induced by methyl-jasmonate. J. Amer. Soc. Hort. Sci. 125:547-552.

Howell, G.S., B.G. Stergios, S.S. Stackhouse, H.C. Bittenbender, and C.L. Burton. 1976. Ethephon as a mechanical harvesting aid for highbush blueberries (Vaccinium austral Small). J. Amer. Soc. Hort. Sci. 101:111-115.

Kazan, K. and J.M. Manners. 2008. Jasmonate signaling: Toward an integrated view. Plant Physiol. 146:1459-1468.

Kazan, K. and J.M. Manners. 2013. MYC2: The master in action. Mol. Plant 6:686-703.

Kim, J. 2014. Four shades of detachment: Regulation of floral organ abscission. Plant Signal. Behav. 9:e976154.

Kim, J., B. Dotson, C. Rey, J. Lindsey, A.B. Bleecker, B.M. Binder, and S.E. Patterson. 2013. New clothes for the jasmonic acid receptor COI1: Delayed abscission, meristem arrest and apical dominance. PLoS One 8:e60505.

Liljegren, S. 2012. Organ abscission: Exit strategies require signals and moving traffic. Curr. Opin. Plant Biol. 15:670-676.

Mao, L., D. Begum, H.-W. Chuang, M.A. Budiman, E.J. Szymkowiak, E.E. Irish, and R.A. Wing. 2000. JOINTLESS is a MADS-box gene controlling tomato flower abscission zone development. Nature 406:910-913.

Malladi, A., T. Vashisth, and L.K. Johnson. 2012. Ethephon and methyl jasmonate affect fruit detachment in rabbiteye and southern highbush blueberry. HortScience 47:1745-1749.

Malladi, A., T. Vashisth, and S. NeSmith. 2013. Development and evaluation of a portable, handheld mechanical shaker to study fruit detachment in blueberry. HortScience 48:394-397. 
McKim, S.M., G.E. Stenvik, M.A. Butenko, W. Kristiansen, S.K. Cho, S.R. Hepworth, R.B. Aalen, and G.W. Haughn. 2008. The BLADE$O N-P E T I O L E$ genes are essential for abscission zone formation in Arabidopsis. Development 135:1537-1546.

Meir, S., S. Philosph-Hadas, S. Sundaresan, V.K.S. Selvara, S. Burd, R. Ophor, B. Kochaneck, M.S. Reid, C.-Z. Jiang, and A. Lers. 2010. Microarray analysis of the abscission-related transcriptome in the tomato flower abscission zone in response to auxin depletion. Plant Physiol. 154:1929-1956.

Nakano, T., M. Fujisawa, Y. Shima, and Y. Ito. 2013. Expression profiling of tomato pre-abscission pedicels provides insights into abscission zone properties including competence to respond to abscission signals. BMC Plant Biol. 13:40.

NeSmith, D.S. 2010. 'Suziblue' southern highbush blueberry. HortScience 45:142-143.

Parra, R., M.A. Paredes, I.M. Sanchez-Calle, and M.C. Gomez-Jimenez. 2013. Comparative transcriptional profiling analysis of olive ripe-fruit pericarp and abscission zone tissues shows expression differences and distinct patterns of transcriptional regulation. BMC Plant Biol. 14:866.

Patterson, S.E. 2001. Cutting loose. Abscission and dehiscence in Arabidopsis. Plant Physiol. 126:494-500.

Pfaffl, M.W. 2001. A new mathematical model for relative quantification in real-time RT-PCR. Nucleic Acids Res. 29:e45.

Ramakers, C., J.M. Ruijter, R.H.L. Deprez, and A.F. Moorman. 2003. Assumption-free analysis of quantitative real-time polymerase chain reaction (PCR) data. Neurosci. Lett. 339:62-66.

Rieu, I. and S.J. Powers. 2009. Real-time quantitative RT-PCR: Design, calculations, and statistics. Plant Cell 21:1031-1033.

Roberts, J.A., K.A. Elliott, and Z.H. Gonzalez-Carranza. 2002. Abscission, dehiscence, and other cell separation processes. Annu. Rev. Plant Biol. 53:131-158.

Ruijter, J.M., C. Ramakers, W.M.H. Hoogaars, Y. Karlen, O. Bakker, M.J.B. van den Hoff, and A.F.M. Moorman. 2009. Amplification efficiency: Linking baseline and bias in the analysis of quantitative PCR data. Nucleic Acids Res. 37:e45.
Sexton, R. and J.A. Roberts. 1982. Cell biology of abscission. Annu. Rev. Plant Physiol. 33:133-162.

Sun, Y., X.-Y. Fan, D.-M. Cao, K. He, W. Tang, J.-Y. Zhu, J.-X. He, M.-Y. Bai, S. Zhu, E. Oh, S. Patil, T.-W. Kim, H. Ji, W.H. Wong, S.Y. Rhee, and Z.-Y. Wang. 2010. Integration of brassinosteroid signal transduction with the transcription network for plant growth regulation in Arabidopsis. Dev. Cell 19:765-777.

Takeda, F., G. Krewer, E.L. Andrews, B. Mullinix, Jr., and C.L. Peterson. 2008. Assessment of v45 over-the-row mechanical harvester on pruned rabbiteye and southern highbush blueberry. HortTechnology 18:130-138.

Taylor, J.E. and C.A. Whitelaw. 2001. Signals in abscission. New Phytol. 151:323-339.

U.S. Department of Agriculture. 1995. United States standards for grades of blueberries. U.S. Dept. Agr., Agr. Mktg. Serv., Washington, DC.

Vashisth, T. and A. Malladi. 2013. Fruit detachment in rabbiteye blueberry: Abscission and physical separation. J. Amer. Soc. Hort. Sci. 138:95-101.

Vashisth, T. and A. Malladi. 2014. Fruit abscission in rabbiteye blueberry in response to organ removal and mechanical wounding. HortScience 49:1403-1407.

Vashisth, T., L.K. Johnson, and A. Malladi. 2011. An efficient RNA isolation procedure and identification of reference genes for normalization of gene expression in blueberry. Plant Cell Rep. 30:21672176.

Wasternack, C. 2007. Jasmonates: An update on biosynthesis, signal transduction and action in plant stress response, growth and development. Ann. Bot. (Lond.) 100:681-697.

Wolf, S., J. Mravec, S. Greiner, G. Mouille, and H. Höfte. 2012. Plant cell wall homeostasis is mediated by brassinosteroid feedback signaling. Curr. Biol. 22:1732-1737.

Yin, Y., D. Vafeados, Y. Tao, S. Yoshida, T. Asami, and J. Chory. 2005. A new class of transcription factors mediates brassinosteroidregulated gene expression in Arabidopsis. Cell 120:249-259. 


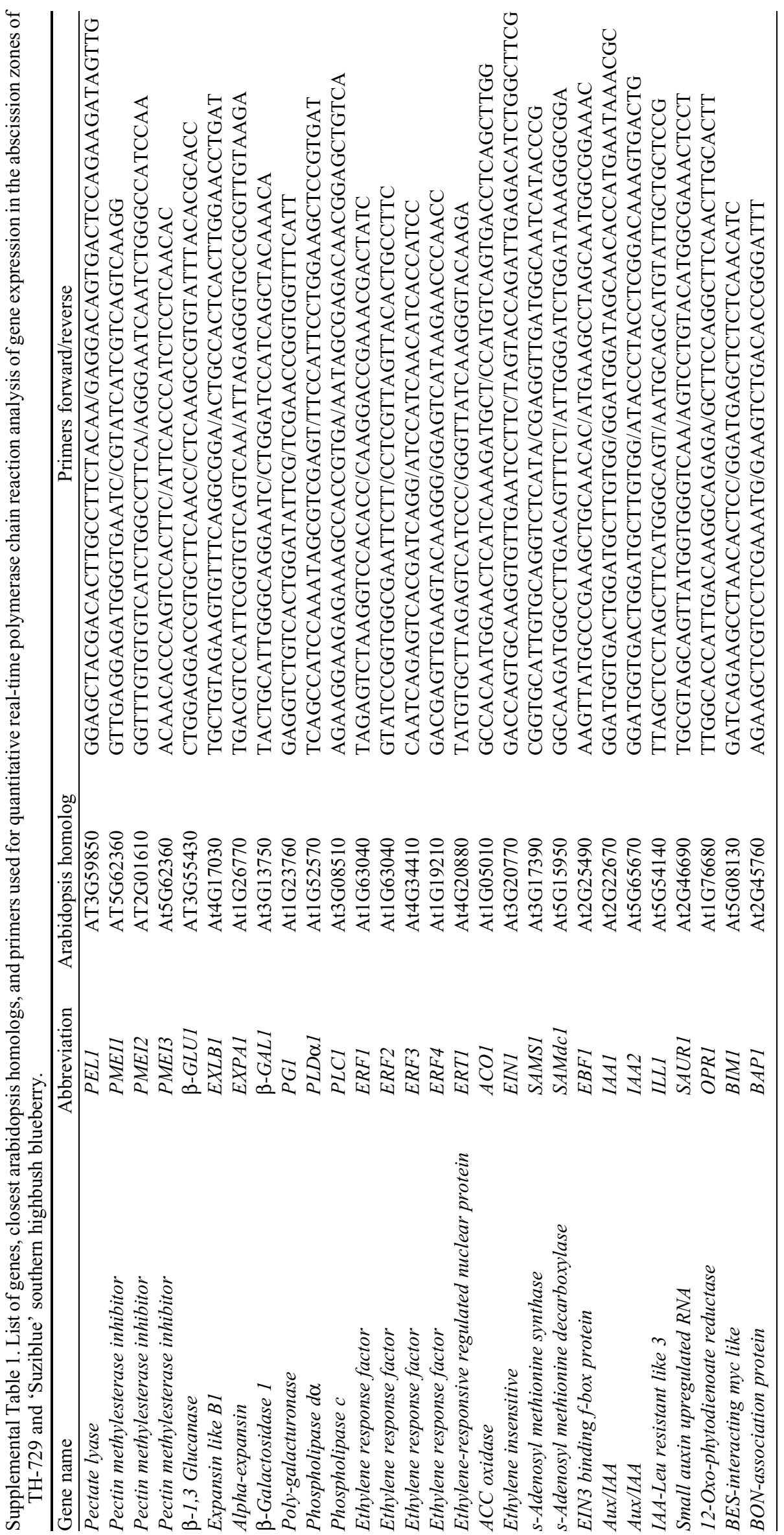




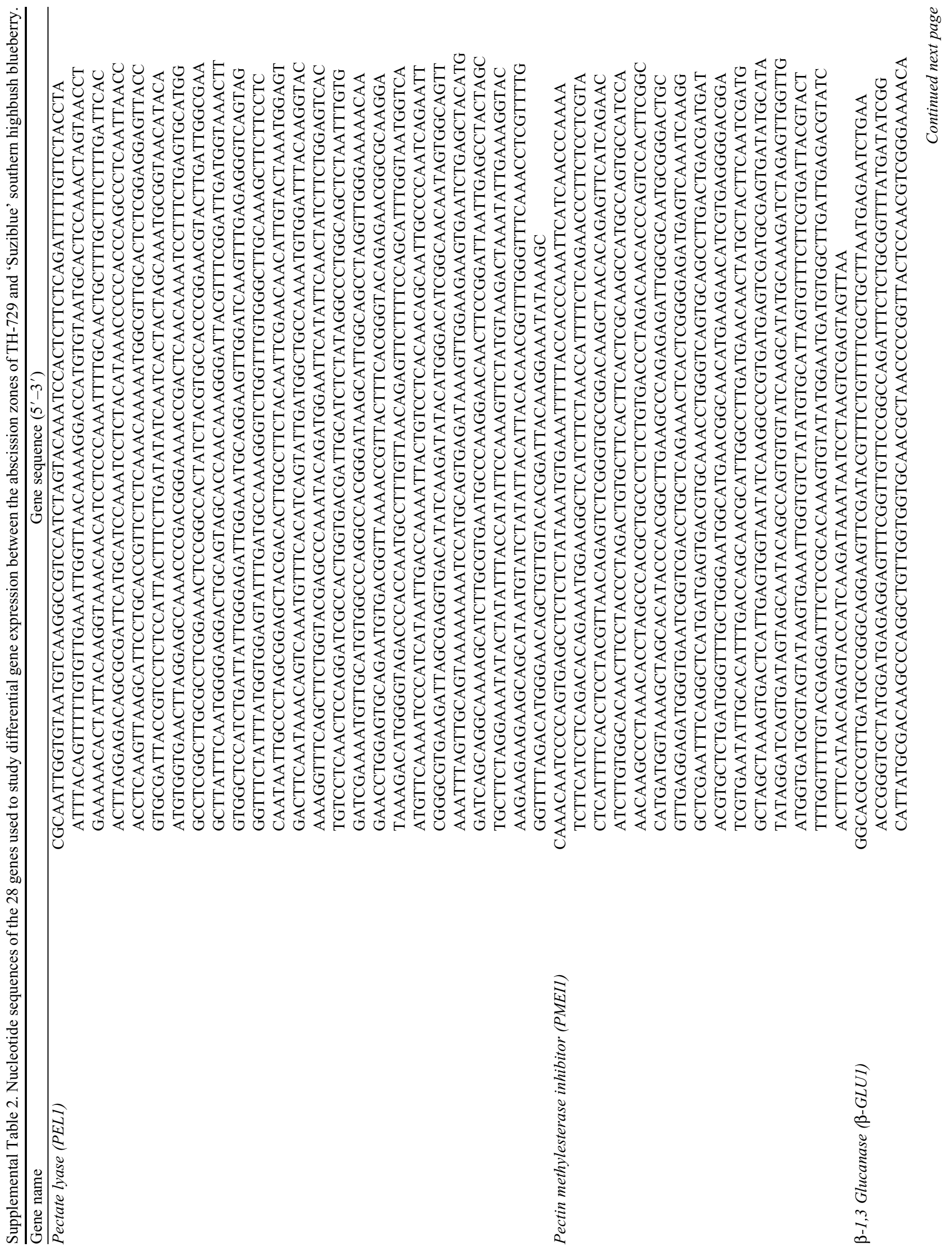




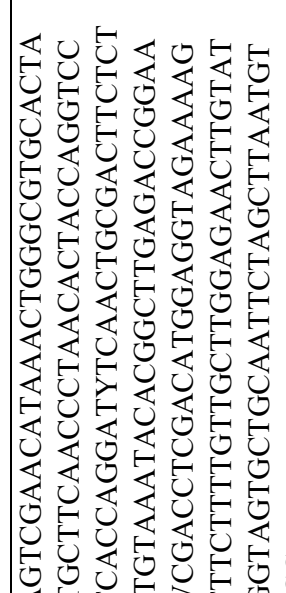

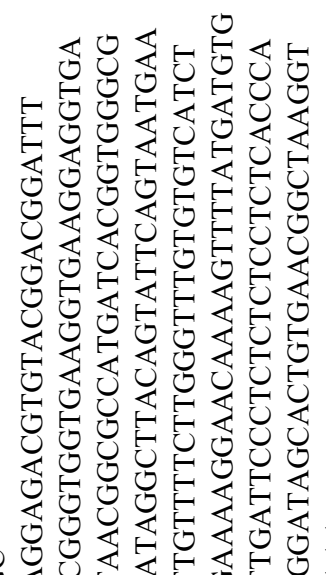

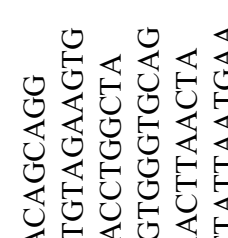

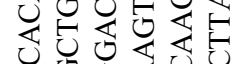

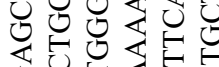

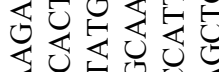

出它记导出

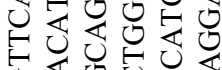

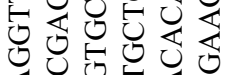

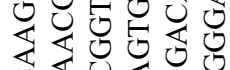

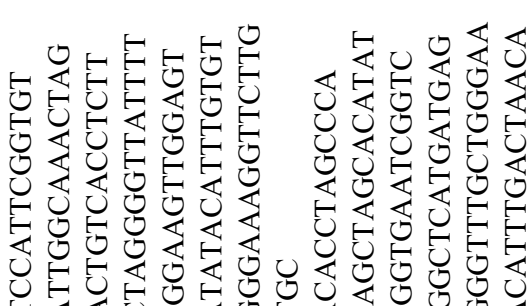

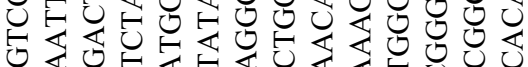

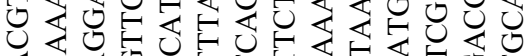

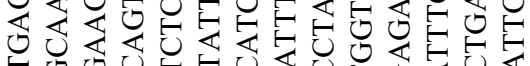

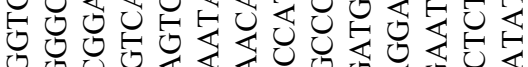

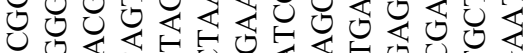

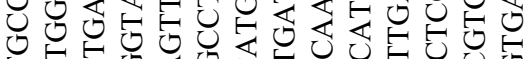
O O

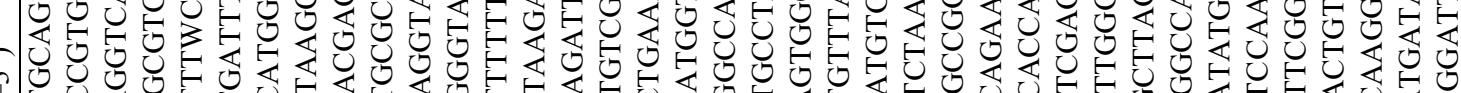
同

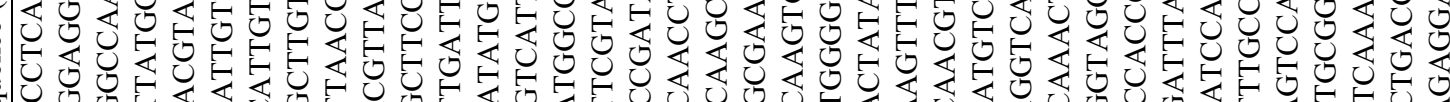

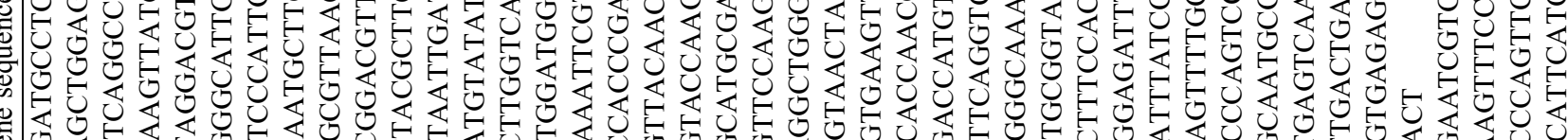

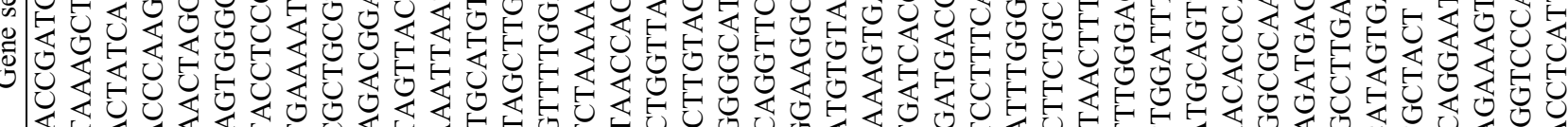

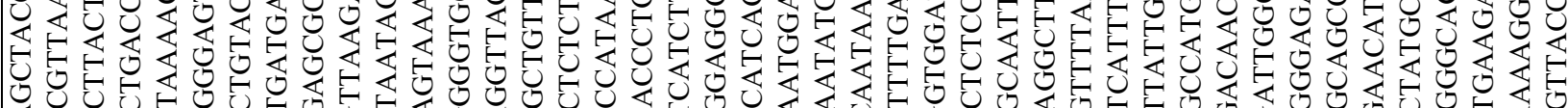

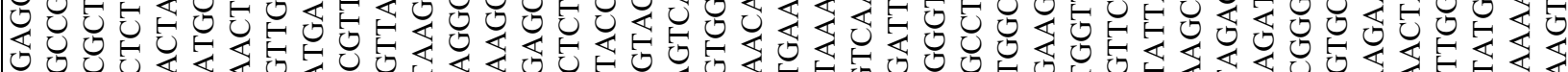
O O O

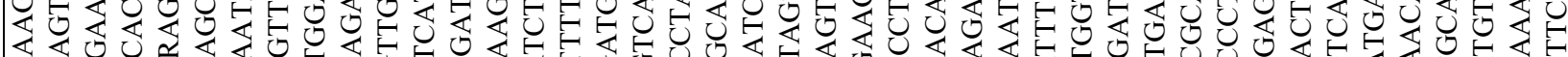

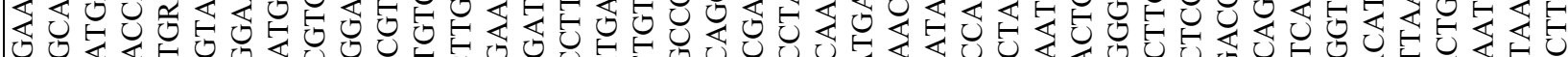

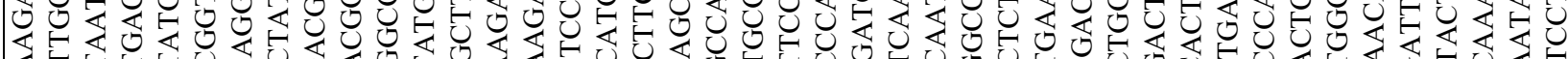

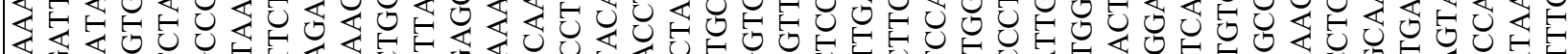

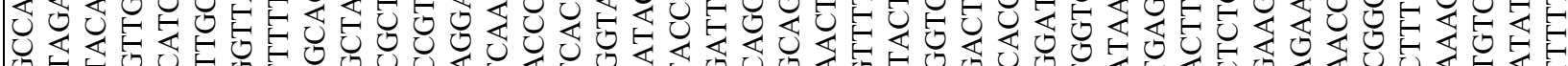
O O

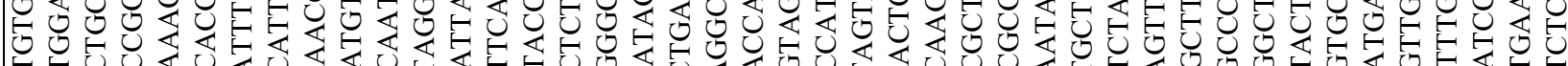

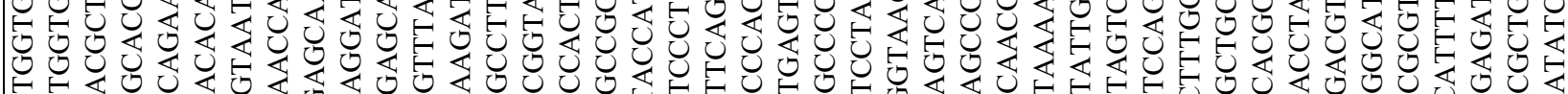

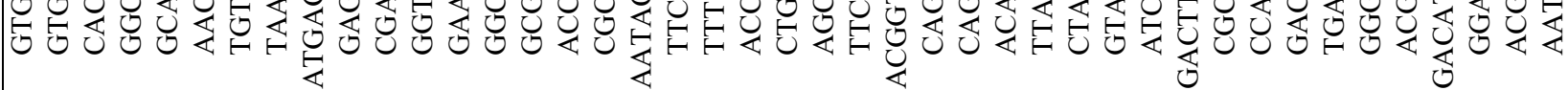

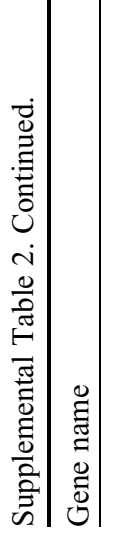

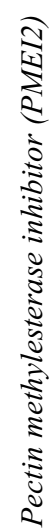

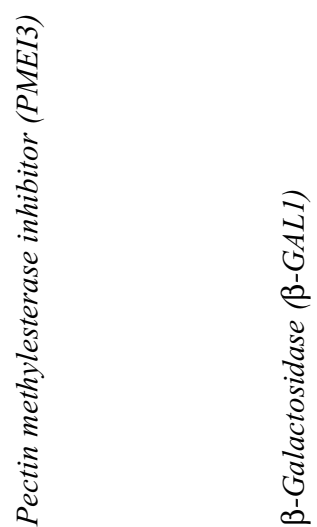




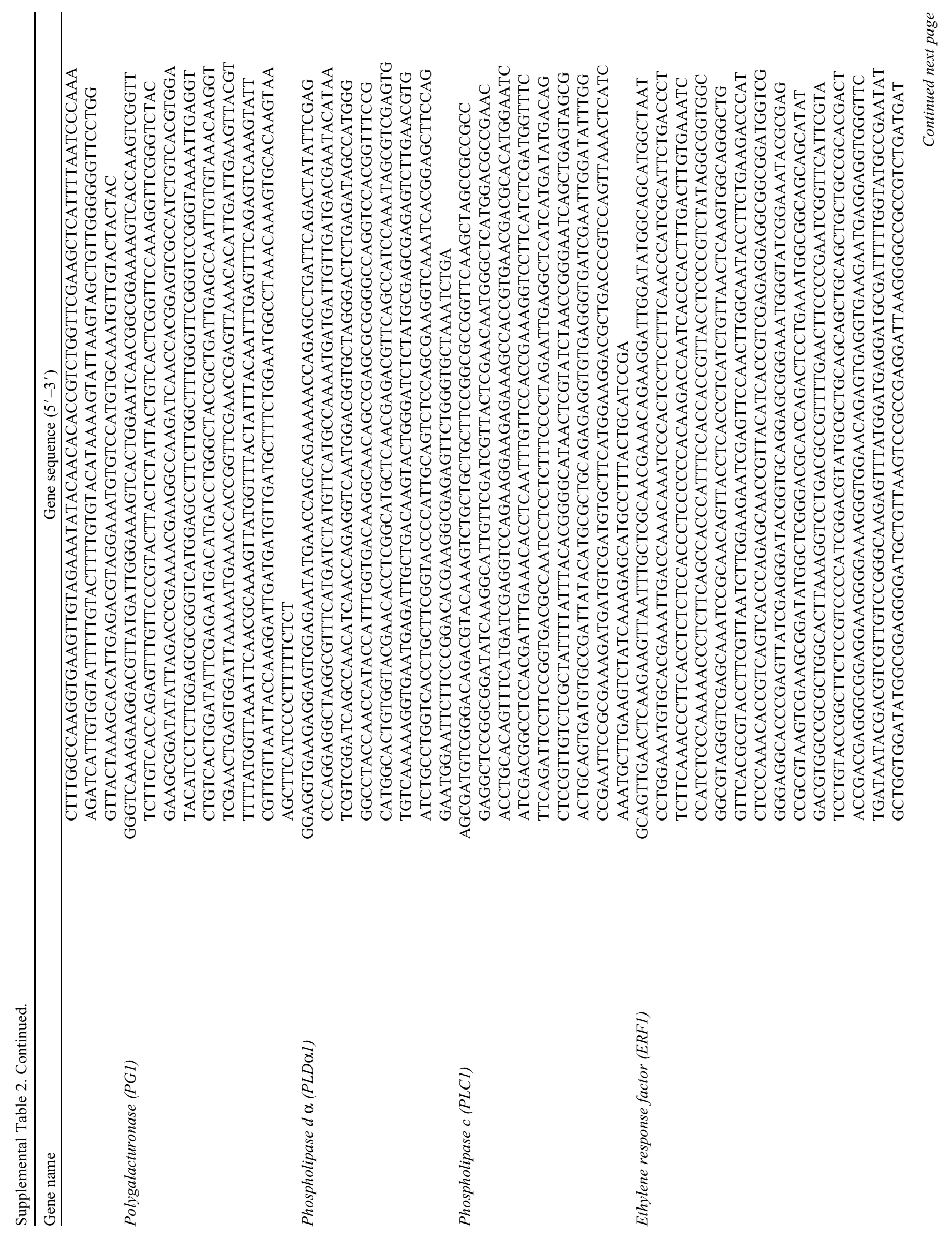




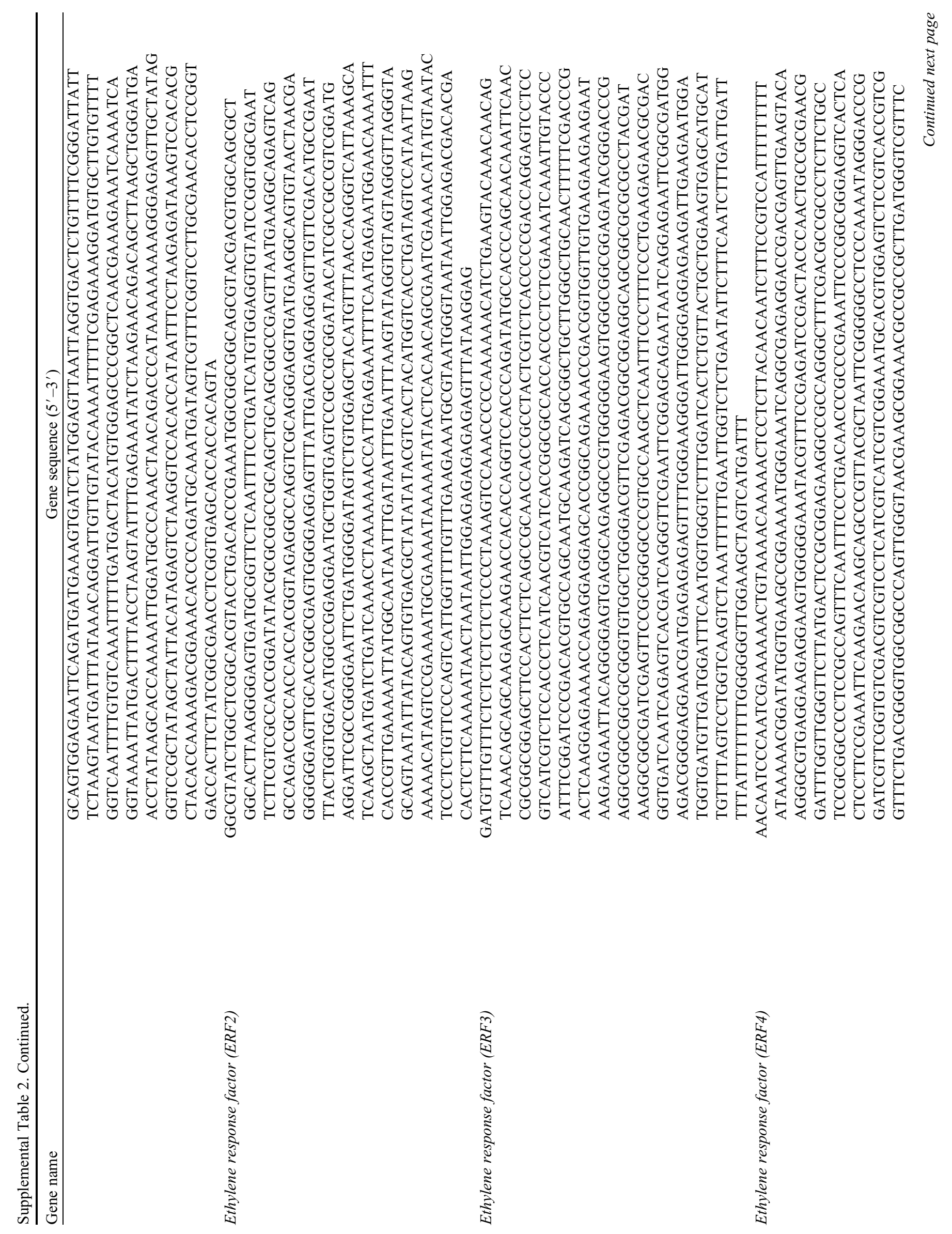




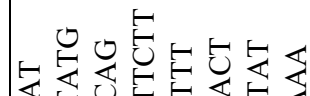

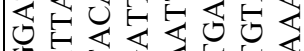
过出出

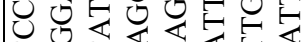

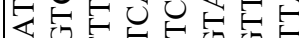
$\Leftrightarrow$

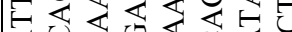

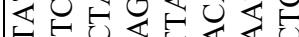

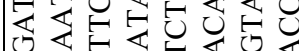
-

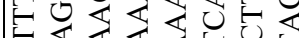

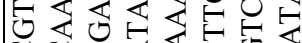
U U O

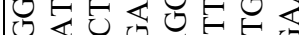
O U U O 论 O

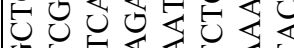

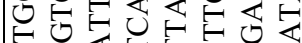
$\varangle$ O

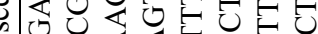

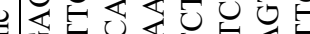

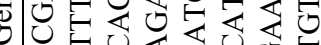
U

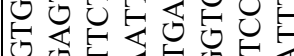
O

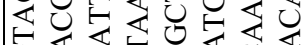

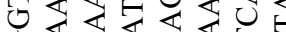
O仓 H

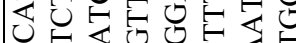

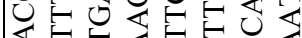

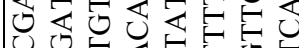
O O

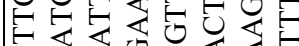

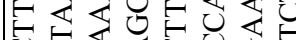

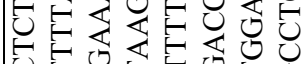
U.

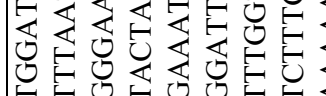
F O

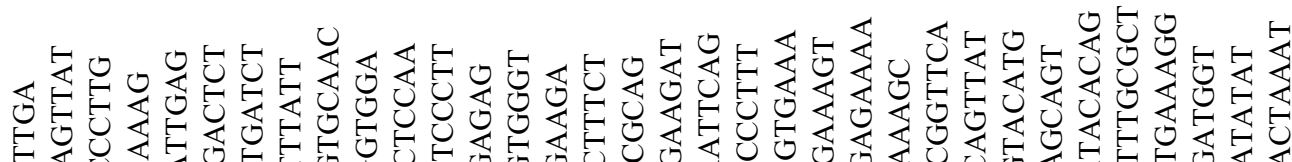

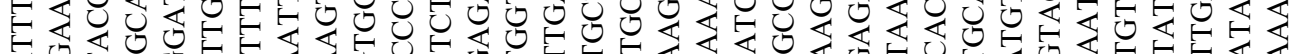

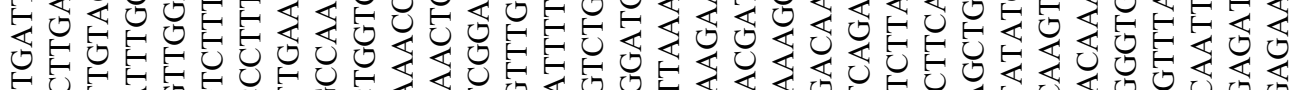

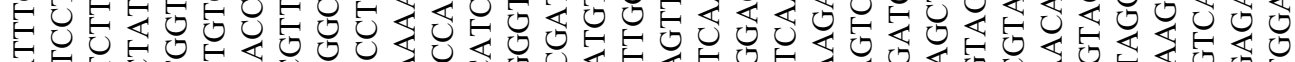

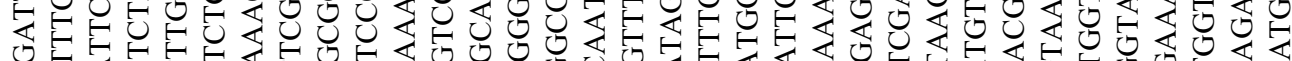

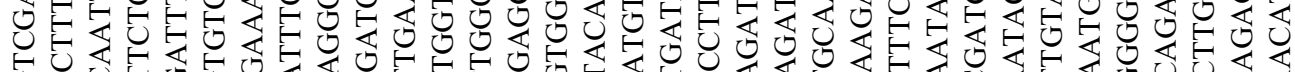

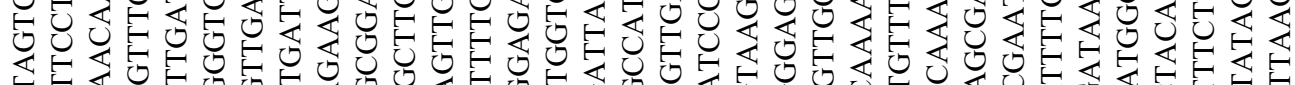

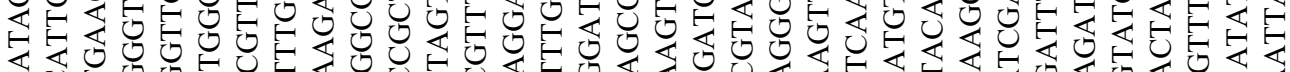

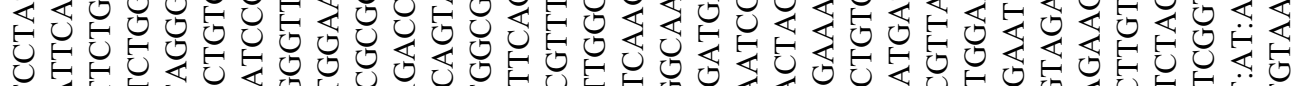

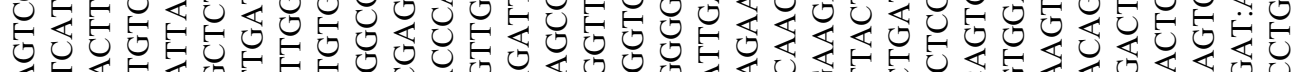

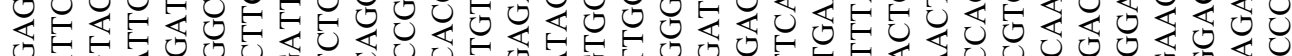

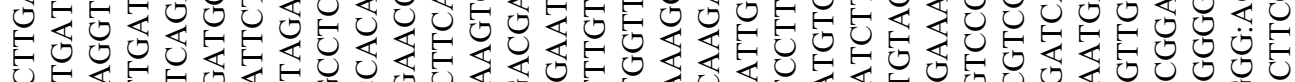

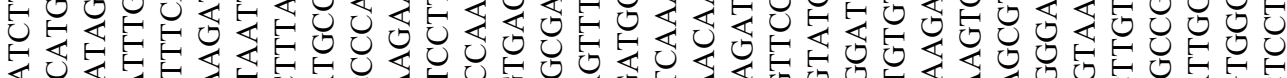

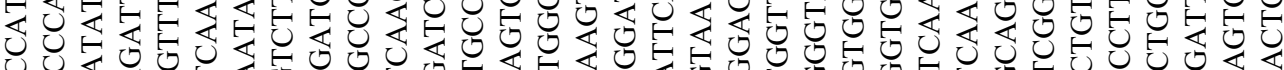
O

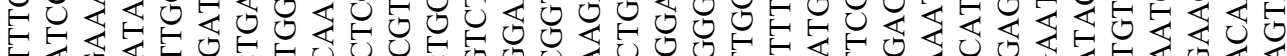

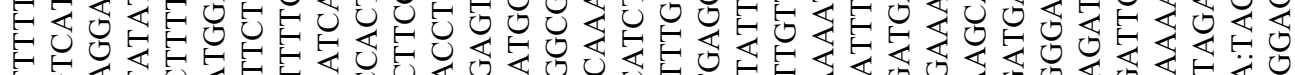

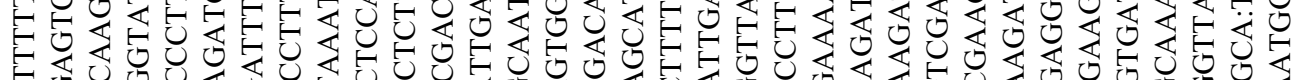

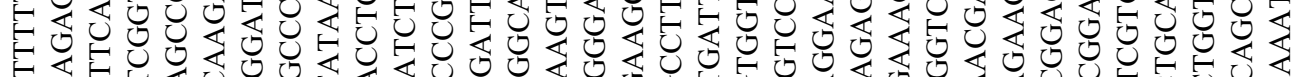

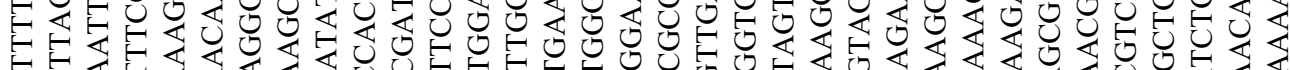

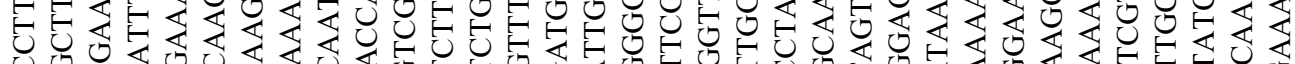

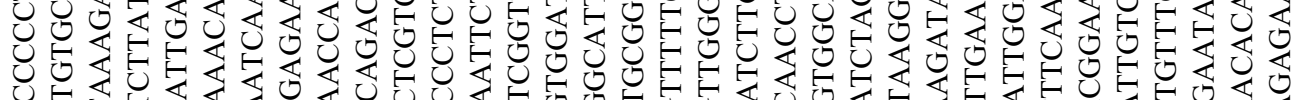

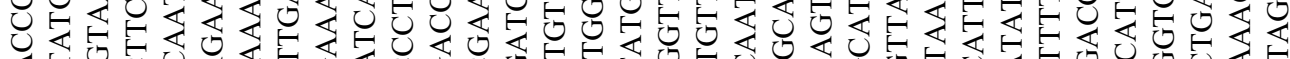

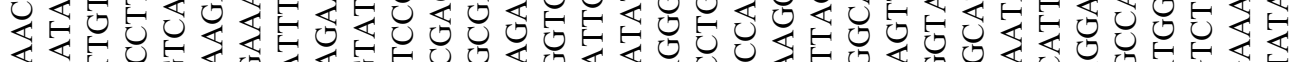

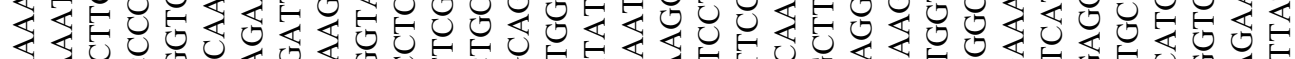

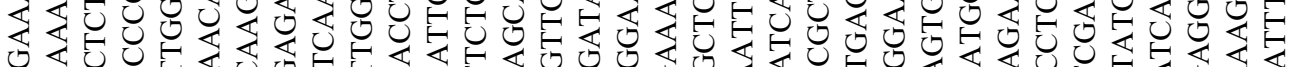

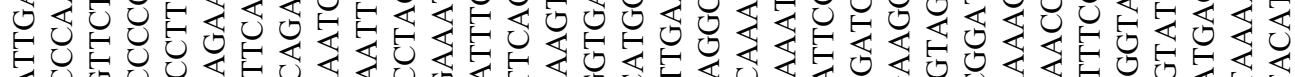

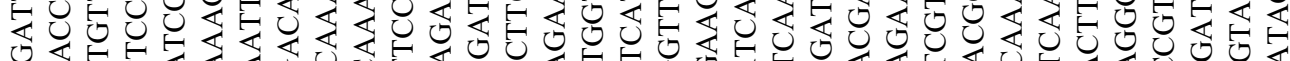

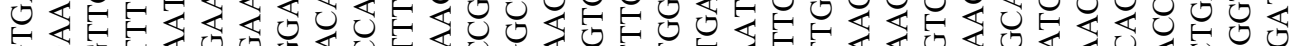

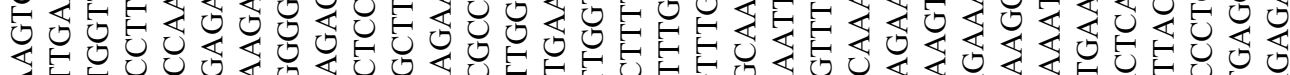

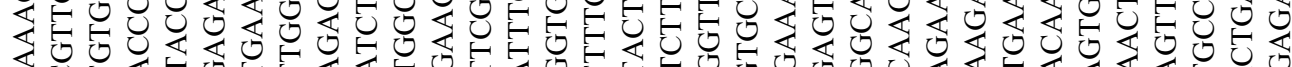

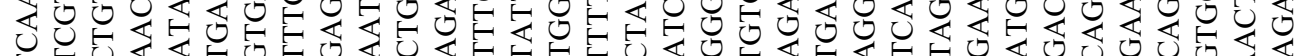

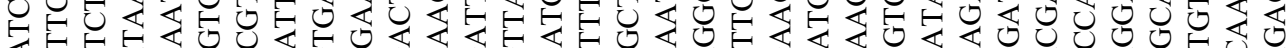
出 


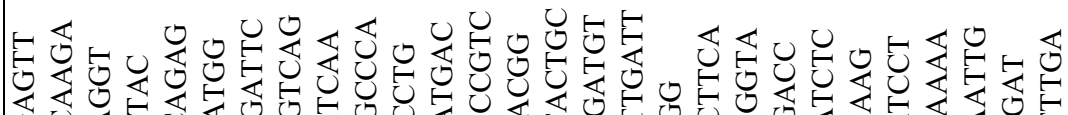

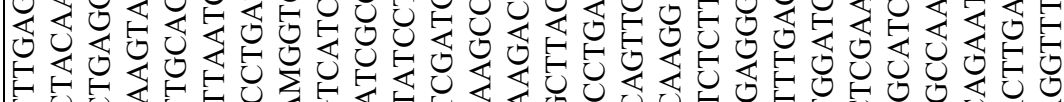

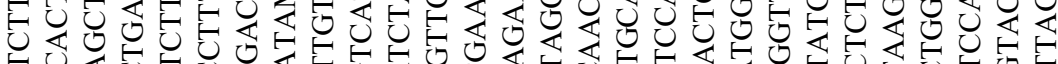

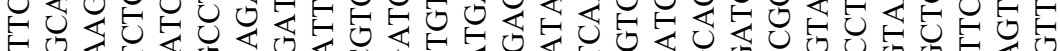

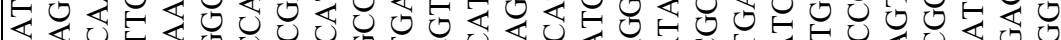
出过

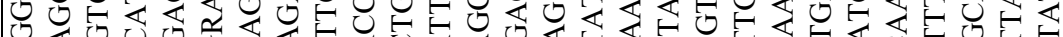
出

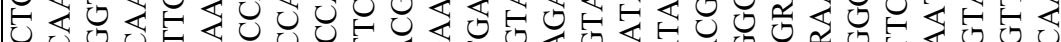

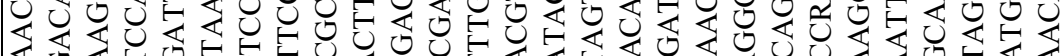

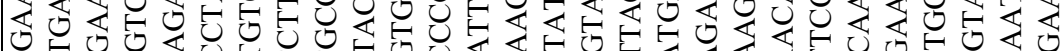

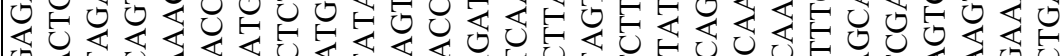

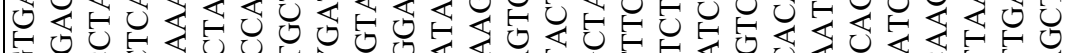
Ð 列 40

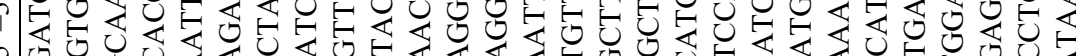

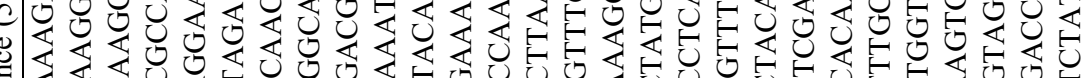
过

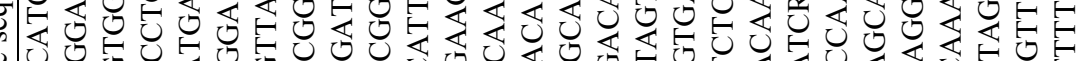
U

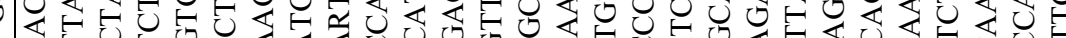

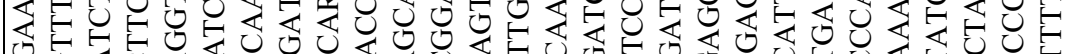
o 0 <

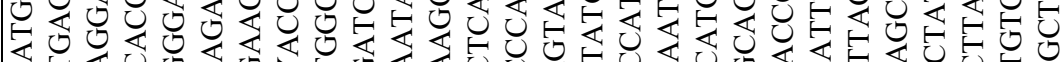

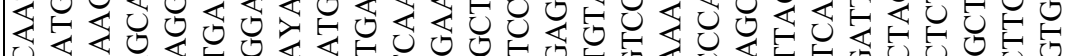

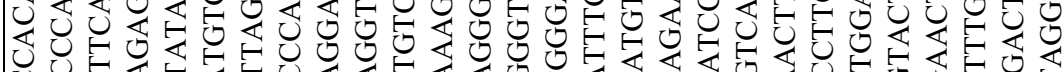
U U

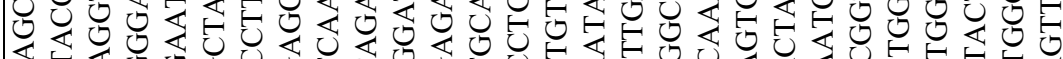

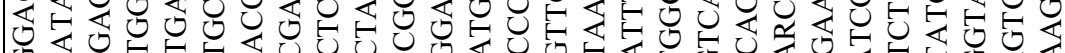

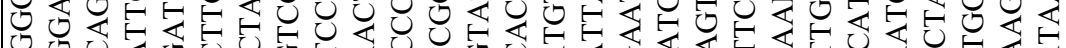

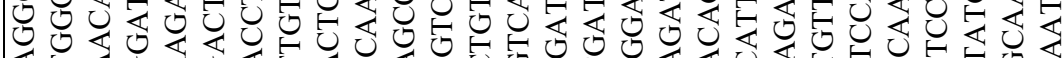

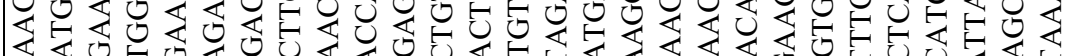

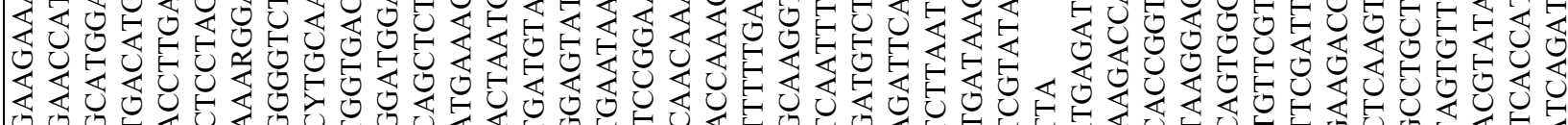

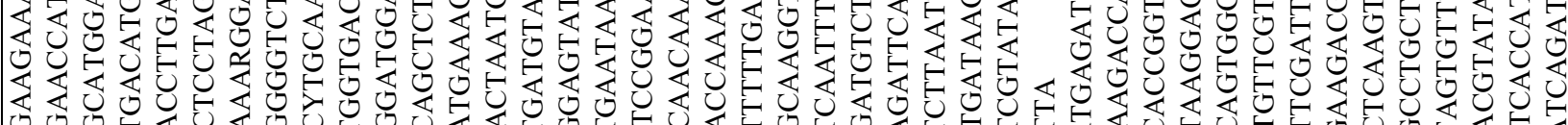
过 出 
《U U U U U U U

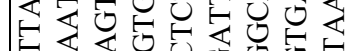

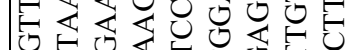

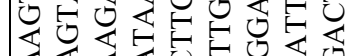

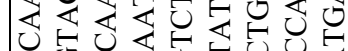

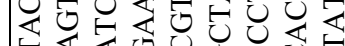

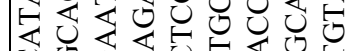
U U

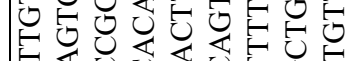

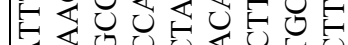

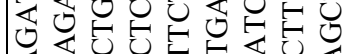

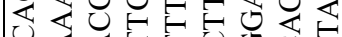

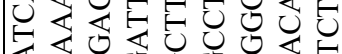
U⿺辶寸 0 U

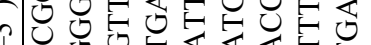

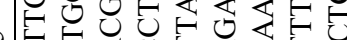

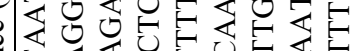

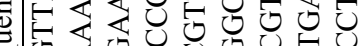

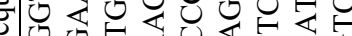

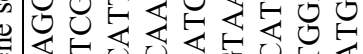
४⿻ 讨出

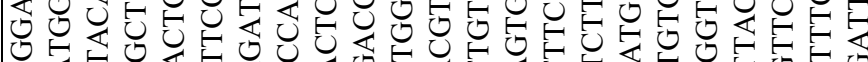
O

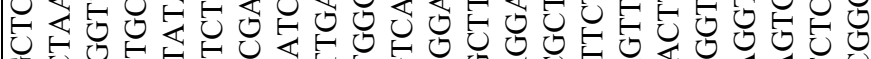

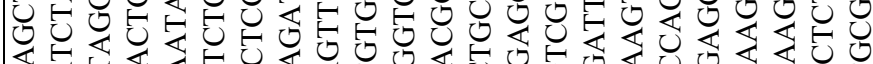

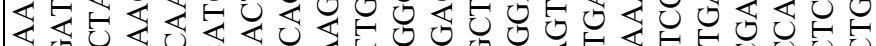
《O

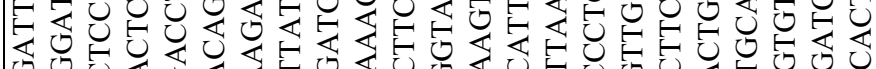

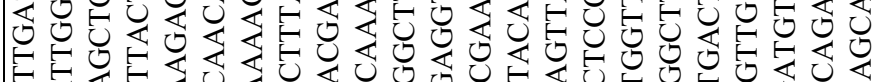

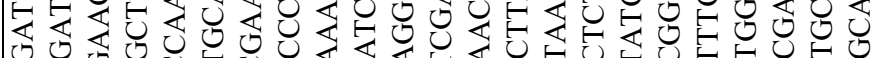
U.

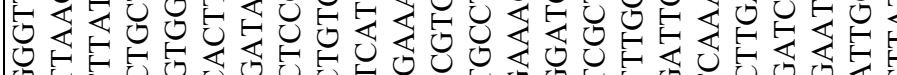

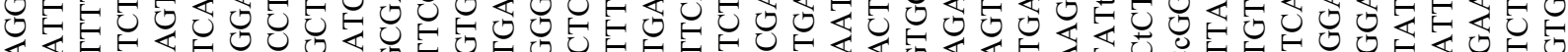

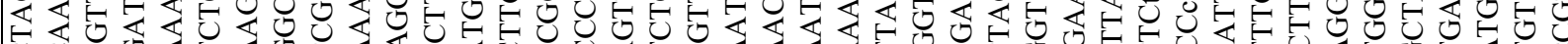

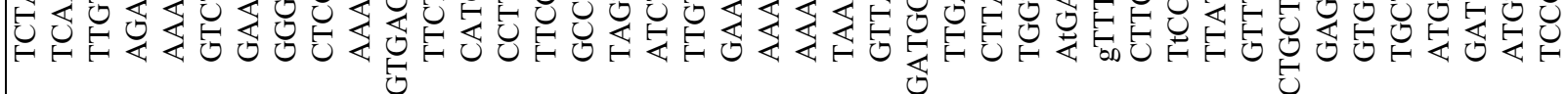

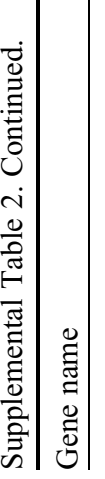

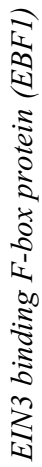




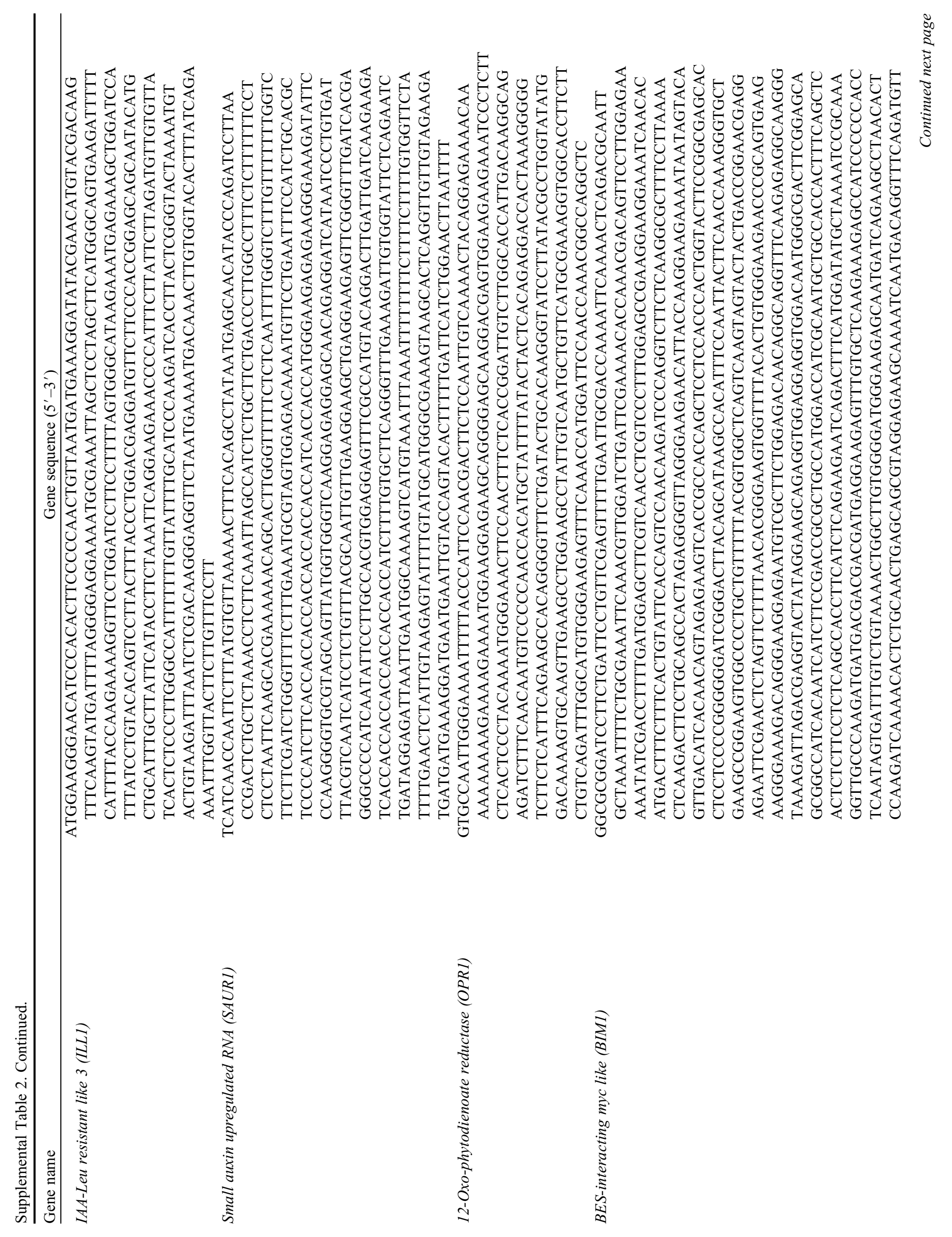




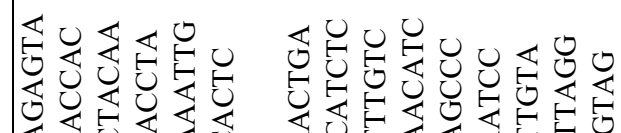

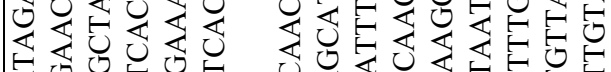

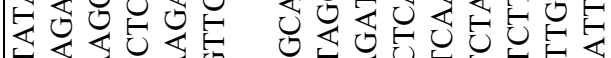

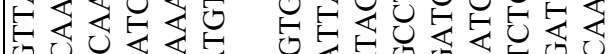

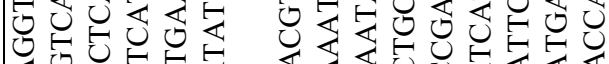

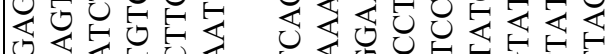

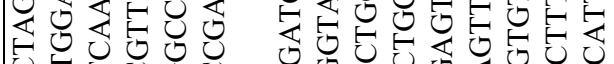
式导记 O

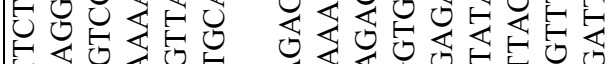

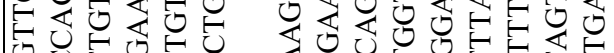
Ư

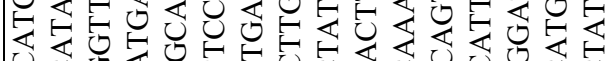

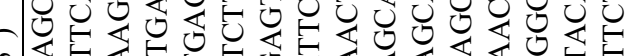
के

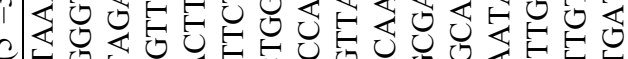

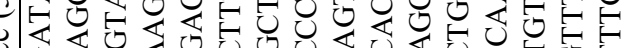

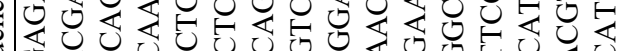

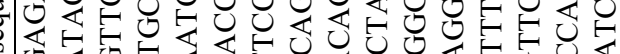

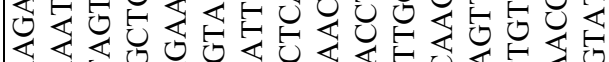

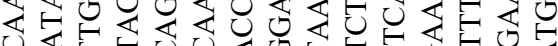

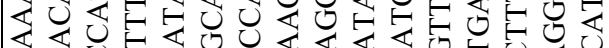

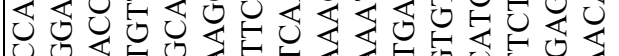

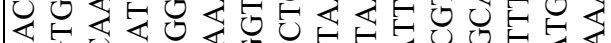

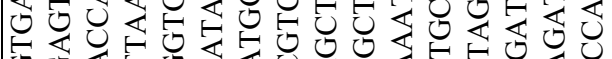

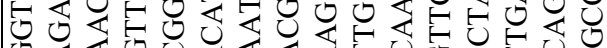

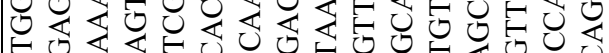

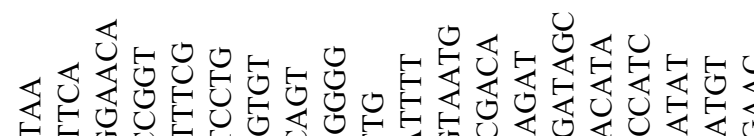

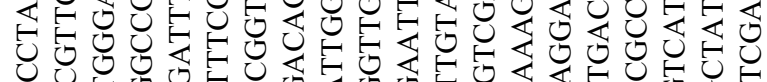

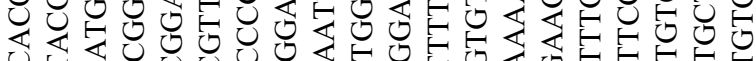

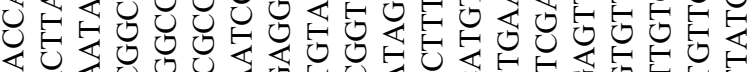

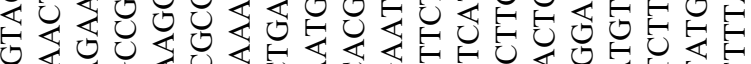

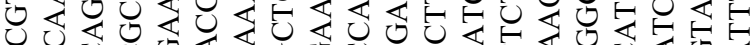
O

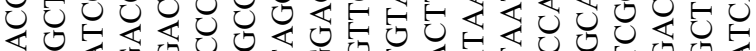

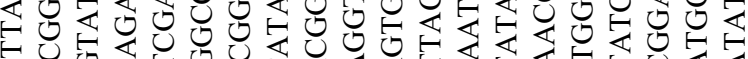

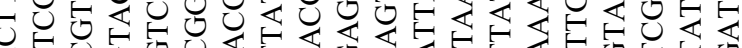

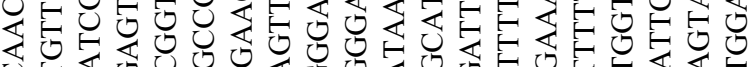

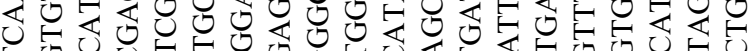

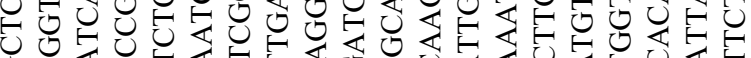

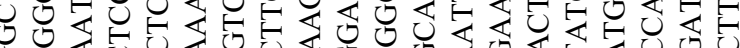
O

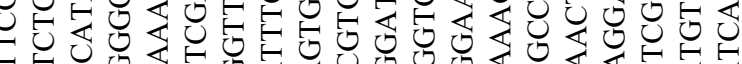

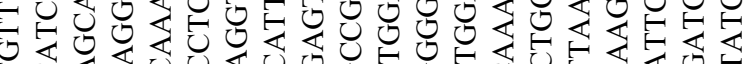

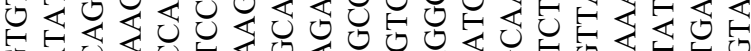

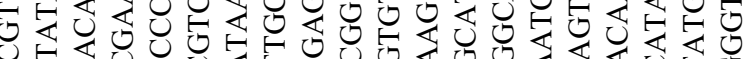

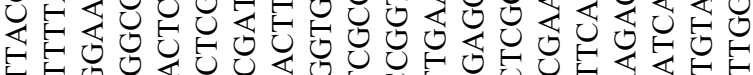
HE

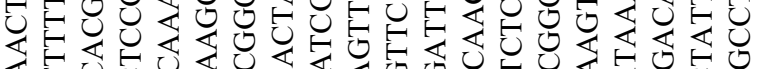

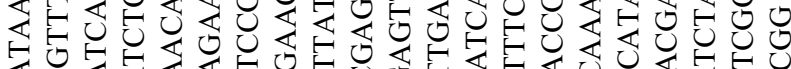

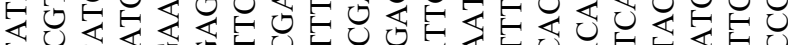

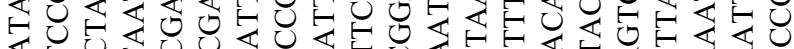

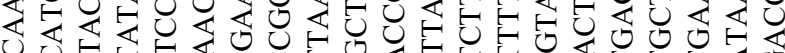

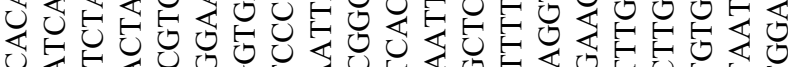

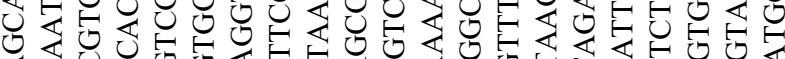

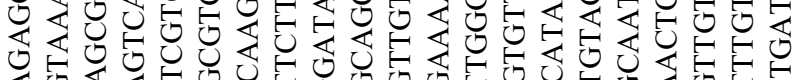

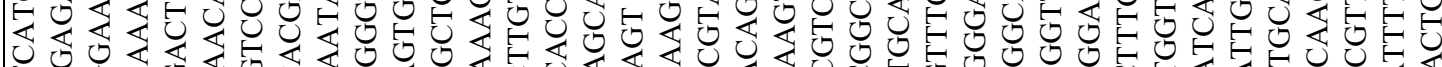

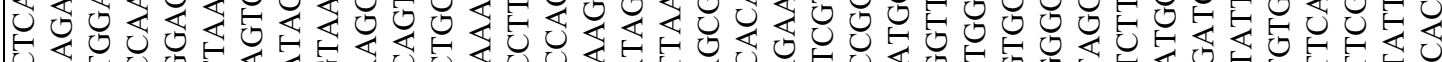
U⿺辶寸

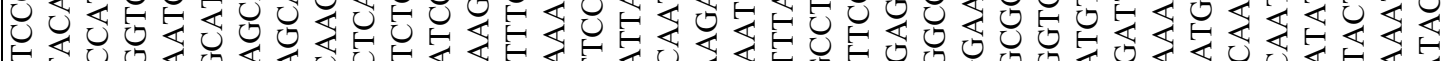

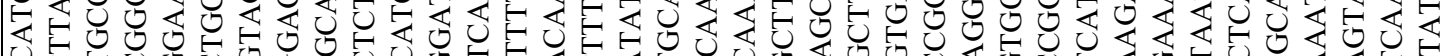
UE

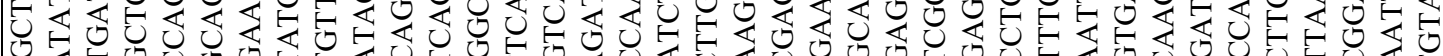

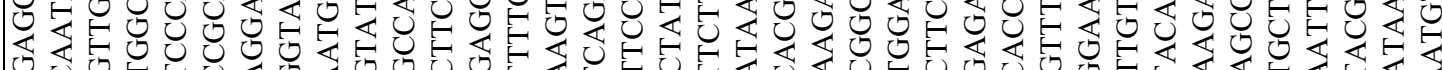
O U

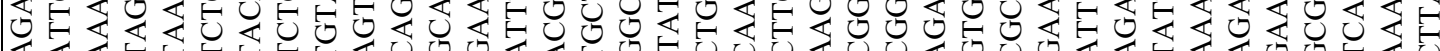

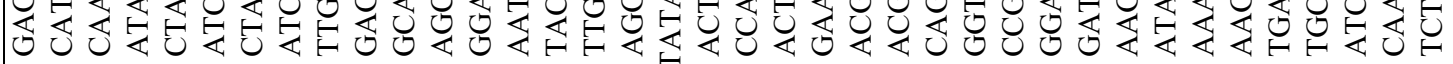

